\title{
Second Order Asymptotic Development for the Anisotropic Cahn-Hilliard Functional
}

\author{
Gianni Dal Maso \\ SISSA, \\ Via Bonomea 265, 34136 Trieste, Italy \\ Irene Fonseca \\ Department of Mathematical Sciences, \\ Carnegie Mellon University, \\ Pittsburgh PA 15213-3890, USA \\ Giovanni Leoni \\ Department of Mathematical Sciences, \\ Carnegie Mellon University, \\ Pittsburgh PA 15213-3890, USA
}

June 8, 2021

\begin{abstract}
The asymptotic behavior of an anisotropic Cahn-Hilliard functional with prescribed mass and Dirichlet boundary condition is studied when the parameter $\varepsilon$ that determines the width of the transition layers tends to zero. The double-well potential is assumed to be even and equal to $|s-1|^{\beta}$ near $s=1$, with $1<\beta<2$. The first order term in the asymptotic development by $\Gamma$-convergence is well-known, and is related to a suitable anisotropic perimeter of the interface. Here it is shown that, under these assumptions, the second order term is zero, which gives an estimate on the rate of convergence of the minimum values.
\end{abstract}

Keywords: Gamma-convergence, Cahn-Hilliard functional, phase transitions. MSC2010: 49J45, 49Q20, 35B25.

\section{Introduction}

In this paper we study the second order term in the asymptotic development by $\Gamma$-convergence for the anisotropic Cahn-Hilliard functional (see, e.g., [20], [15], [21], 26], [14, [6], 4])

$$
\mathcal{W}_{\varepsilon}(u):=\int_{\Omega}\left(W(u(x))+\varepsilon^{2} \Phi^{2}(\nabla u(x))\right) d x,
$$


where $\Omega$ is a bounded open set in $\mathbb{R}^{n}, n \geq 2$, with Lipschitz boundary. Here $W: \mathbb{R} \rightarrow[0,+\infty)$ is an even function of class $C^{1}$ such that $W(s)=0$ if and only if $s= \pm 1$, with $W(s)=|s-1|^{\beta}$ near $s=1$ for some $1<\beta<2$, and $\Phi: \mathbb{R}^{n} \rightarrow[0,+\infty)$ is convex, even, and positively homogeneous of degree one.

We impose a mass constraint and a boundary condition:

$$
u \in H^{1}(\Omega), \quad \int_{\Omega} u(x) d x=m, \quad \text { and } \quad u=1 \text { on } \partial \Omega,
$$

where $m$ is a prescribed constant satisfying the inequalities

$$
-|\Omega|<m<|\Omega| .
$$

Given a sequence of functionals $F_{\varepsilon}: X \rightarrow(-\infty, \infty]$ defined on a metric space $X$, we write $F_{\varepsilon} \stackrel{\Gamma}{\rightarrow} F^{(0)}$ if $\left\{F_{\varepsilon}\right\} \Gamma$-converges to $F^{(0)}$, as $\varepsilon \rightarrow 0+$, with respect to the metric topology of $X$. We recall the notion of asymptotic development by $\Gamma$-convergence of order $k$ :

$$
F_{\varepsilon} \stackrel{\Gamma}{=} F^{(0)}+\varepsilon F^{(1)}+\cdots+\varepsilon^{k} F^{(k)}+o\left(\varepsilon^{k}\right)
$$

if $F_{\varepsilon} \stackrel{\Gamma}{\rightarrow} F^{(0)}$ and

$$
F_{\varepsilon}^{(i)}:=\frac{F_{\varepsilon}^{(i-1)}-\inf _{X} F^{(i-1)}}{\varepsilon} \stackrel{\Gamma}{\rightarrow} F^{(i)}
$$

for $i=1, \ldots, k$, where $F_{\varepsilon}^{(0)}:=F_{\varepsilon}$ (see [2], [3], [8, Section 1.10]).

For the sequence of functionals (1.1) we take $X:=L^{1}(\Omega)$ and we set $\mathcal{W}_{\varepsilon}(u):=+\infty$ if (1.2) is not satisfied. The zero order term is

$$
\mathcal{W}^{(0)}(u):=\int_{\Omega} W(u(x)) d x
$$

if the mass constraint in (1.2) is satisfied and $\mathcal{W}^{(0)}(u):=+\infty$ otherwise. The $\Gamma$-liminf inequality is a consequence of Fatou's Lemma. The $\Gamma$-limsup inequality is straightforward.

Note that $\inf _{X} \mathcal{W}^{(0)}=0$ and the minimizers are given by all functions of the form $u_{E}:=1-2 \chi_{E}$, where $E$ is an arbitrary measurable subset of $\Omega$ satisfying the volume constraint

$$
|E|=\frac{|\Omega|-m}{2}=: V_{m},
$$

which is equivalent to the mass constraint in (1.2) for $u_{E}$. Here, and in what follows, $\chi_{E}$ is the characteristic function of $E$ defined by $\chi_{E}:=1$ on $E$ and $\chi_{E}:=0$ on $\Omega \backslash E$

To study the first order term for (1.1), we introduce the rescaled functionals defined by

$$
\mathcal{F}_{\varepsilon}(u):=\int_{\Omega}\left(\frac{1}{\varepsilon} W(u(x))+\varepsilon \Phi^{2}(\nabla u(x))\right) d x
$$

if (1.2) is satisfied. We extend $\mathcal{F}_{\varepsilon}$ to $L^{1}(\Omega)$ by setting $\mathcal{F}_{\varepsilon}(u):=+\infty$ if (1.2) is not satisfied. 
By adapting well-known arguments developed in [4, [6], [20, [21], [26], it can be shown (see Theorem 2.1 below) that the first order term $\mathcal{W}^{(1)}$ for (1.1), which by (1.4) coincides with the $\Gamma$-limit of $\left\{\mathcal{F}_{\varepsilon}\right\}$, is given by

$$
\mathcal{F}_{0}(u):=c_{W} \mathrm{P}_{\Phi}(E)
$$

if

$$
u=u_{E}:=1-2 \chi_{E}, \quad E \subset \Omega, \quad \mathrm{P}(E)<+\infty, \text { and } \quad|E|=V_{m},
$$

while $\mathcal{F}_{0}(u):=+\infty$ if (1.7) is not satisfied. Here

$$
c_{W}:=2 \int_{-1}^{1} \sqrt{W(s)} d s
$$

and $\mathrm{P}_{\Phi}$ is the $\Phi$-perimeter, defined for every $E \subset \mathbb{R}^{n}$ with finite perimeter by

$$
\mathrm{P}_{\Phi}(E):=\int_{\partial^{*} E} \Phi\left(\nu_{E}(x)\right) d \mathcal{H}^{n-1}(x),
$$

where $\partial^{*} E$ is the reduced boundary of $E, \nu_{E}$ is the measure theoretic outer unit normal of $E$, and $\mathcal{H}^{n-1}$ is the $(n-1)$-dimensional Hausdorff measure. Observe that in contrast with the results in the literature just quoted, due to the boundary condition in (1.2) in (1.6) we obtain the full $\Phi$-perimeter of $E$ as opposed to the relative $\Phi$-perimeter of $E$ in $\Omega$.

The main goal of this paper is to study the second order term $\mathcal{W}^{(2)}$ for (1.1). Under some additional assumptions on $\Omega$ and $W$ (see (2.2), (2.3), (2.20), and (2.21) in Section 2), we prove that $\mathcal{W}^{(2)}(u)=0$ if $u$ is a minimizer of $\mathcal{F}_{0}$ and $\mathcal{W}^{(2)}(u)=+\infty$ otherwise. The second assertion is trivial. By (1.4) the first assertion amounts to proving the following properties:

(a) ( $\Gamma$-liminf inequality) for every sequence $\left\{u_{\varepsilon}\right\} \subset H^{1}(\Omega)$ satisfying (1.2) and converging strongly in $L^{1}(\Omega)$ to a minimizer $u_{0}$ of $\mathcal{F}_{0}$, we have

$$
\liminf _{\varepsilon \rightarrow 0+} \frac{\mathcal{F}_{\varepsilon}\left(u_{\varepsilon}\right)-\mathcal{F}_{0}\left(u_{0}\right)}{\varepsilon} \geq 0
$$

(b) ( $\Gamma$-limsup inequality) for every minimizer $u_{0}$ of $\mathcal{F}_{0}$ there exists a sequence $\left\{u_{\varepsilon}\right\} \subset H^{1}(\Omega)$ converging strongly to $u_{0}$ in $L^{1}(\Omega)$, satisfying (1.2) and such that

$$
\limsup _{\varepsilon \rightarrow 0+} \frac{\mathcal{F}_{\varepsilon}\left(u_{\varepsilon}\right)-\mathcal{F}_{0}\left(u_{0}\right)}{\varepsilon} \leq 0 .
$$

By standard properties of $\Gamma$-convergence the inequalities (a) and (b) imply that

$$
\min \mathcal{F}_{\varepsilon}=\min \mathcal{F}_{0}+o(\varepsilon)=c_{W} \mathrm{P}_{\Phi}\left(E_{0}\right)+o(\varepsilon),
$$

where $E_{0}$ is a minimizer of $\mathrm{P}_{\Phi}$ under the constraint (1.7), which gives

$$
\min \mathcal{W}_{\varepsilon}=\varepsilon c_{W} \mathrm{P}_{\Phi}\left(E_{0}\right)+o\left(\varepsilon^{2}\right) .
$$


A similar problem was studied in [3] for the single-well potential $W(s)=s^{2}$ without imposing the mass constraint and assuming a strictly positive boundary condition $g$. This forces a transition near $\partial \Omega$ and leads to a second order term $\mathcal{W}^{(2)}$ in the asymptotic expansion of the form $\frac{1}{2} \int_{\partial \Omega} g^{2} K d \mathcal{H}^{n-1}$, where $K$ is the mean curvature of $\partial \Omega$.

We conclude by discussing our hypotheses. The assumption that $W$ is even is used in a crucial way to cancel many terms in the estimates due to symmetry arguments. The hypothesis that $W(s)=|s-1|^{\beta}$ near $s=1$ for some $1<\beta<2$ is also important. Indeed, in the case $\beta=2$ and without assuming the boundary condition in (1.2), it can be shown that the second order term $\mathcal{W}^{(2)}$ in the asymptotic expansion may be different from zero (see [18]).

Finally, we observe that the case $n=1$ is completely different, since the minimizers of $\mathrm{P}_{\Phi}$ under the constraint (1.7) are intervals and so the geometry plays no role. However, different nontrivial issues have been addressed (see, e.g., in [5] and [9], and also [2]).

\section{Preliminaries}

Let $W: \mathbb{R} \rightarrow \mathbb{R}$ be a double well potential of class $C^{1}$ such that $W \geq 0$ and $W(s)=0$ if and only if $s= \pm 1$. Assume, in addition, that

$$
\begin{aligned}
& W(s)=W(-s), \\
& W^{\prime}(s)>0 \text { for } s>1, \\
& \liminf _{s \rightarrow+\infty} W^{\prime}(s)>0,
\end{aligned}
$$

and that there exist two constants $0<a<1$ and $1<\beta<2$ such that

$$
W(s)=|s-1|^{\beta} \quad \text { for } 1-a \leq s \leq 1+a .
$$

Let $z$ be the unique global solution with values in $[-1,1]$ of the Cauchy problem

$$
z^{\prime}(t)=\sqrt{W(z(t))}, \quad z(0)=0 .
$$

A rescaled version of this function will play an important role in the construction of the recovery sequence (see (4.3) ) for the $\Gamma$-limsup inequality (1.11) . For this reason, the function $z$ will be called the "optimal profile" of the phase transition.

For $-1<z(t)<1$ we obtain, by integration,

$$
t=\int_{0}^{z(t)} \frac{d s}{\sqrt{W(s)}}
$$

It follows that $z$ is odd and $z(t)=1$ for all $t \geq \tau_{W}$, where

$$
\tau_{W}:=\int_{0}^{1} \frac{d s}{\sqrt{W(s)}},
$$


which is finite thanks to (2.4) since $\beta<2$. Moreover $-1<z(t)<1$ for $-\tau_{W}<t<\tau_{W}$.

Define

$$
c_{W}:=\int_{-\tau_{W}}^{\tau_{W}}\left(W(z(t))+\left|z^{\prime}(t)\right|^{2}\right) d t .
$$

Note that by (2.5) we have

$$
\int_{-\tau_{W}}^{\tau_{W}} W(z(t)) d t=\int_{-\tau_{W}}^{\tau_{W}}\left|z^{\prime}(t)\right|^{2} d t=\frac{c_{W}}{2},
$$

therefore (1.8) holds. It can be shown that for every $b \geq \tau_{W}$ the function $z$ is the unique solution of the minimum problem

$$
\min _{w \in H^{1}(-b, b), w(0)=0} \int_{-b}^{b}\left(W(w(s))+\left|w^{\prime}(s)\right|^{2}\right) d s=c_{W} .
$$

Let $\Phi: \mathbb{R}^{n} \rightarrow \mathbb{R}, n \geq 2$, be convex, even, and positively homogeneous of degree one, such that

$$
c_{\Phi}|\xi| \leq \Phi(\xi) \leq C_{\Phi}|\xi|
$$

for all $\xi \in \mathbb{R}^{n}$ and for some $C_{\Phi} \geq c_{\Phi}>0$, where $|\cdot|$ is the Euclidean norm in $\mathbb{R}^{n}$.

The polar function $\Phi^{\circ}$ is defined by

$$
\Phi^{\circ}(\eta):=\sup _{\xi \neq 0} \frac{\eta \cdot \xi}{\Phi(\xi)}
$$

for every $\eta \in \mathbb{R}^{n}$. It turns out that $\Phi^{\circ}$ is convex, even, positively homogeneous of degree one on $\mathbb{R}^{n}$ (see [24]), and

$$
\Phi\left(\nabla \Phi^{\circ}(\eta)\right)=1 \quad \text { for a.e. } \eta \in \mathbb{R}^{n} .
$$

Moreover, it satisfies the inequalities

$$
\frac{1}{C_{\Phi}}|\eta| \leq \Phi^{\circ}(\eta) \leq \frac{1}{c_{\Phi}}|\eta|
$$

for every $\eta \in \mathbb{R}^{n}$.

The ball with respect to the norm $\Phi^{\circ}$ centered at $x_{0} \in \mathbb{R}^{n}$ and with radius $\rho>0$ is denoted by

$$
B_{\rho}^{\Phi^{\circ}}\left(x_{0}\right):=\left\{x \in \mathbb{R}^{n}: \Phi^{\circ}\left(x-x_{0}\right)<\rho\right\},
$$

Observe that

$$
\begin{aligned}
\left|B_{\rho}^{\Phi^{\circ}}\left(x_{0}\right)\right| & =\kappa_{\Phi} \rho^{n}, \\
\mathrm{P}_{\Phi}\left(B_{\rho}^{\Phi^{\circ}}\left(x_{0}\right)\right) & =n \kappa_{\Phi} \rho^{n-1},
\end{aligned}
$$


where $\mathrm{P}_{\Phi}$ is the $\Phi$-perimeter introduced in (1.9), and

$$
\kappa_{\Phi}:=\left|B_{1}^{\Phi^{\circ}}(0)\right|
$$

It is easy to check (see, e.g., [1, 12]) that for every measurable function $w$ : $[0, R] \rightarrow[0,+\infty]$ we have

$$
\int_{B_{R}^{\Phi^{\circ}}\left(x_{0}\right)} w\left(\Phi^{\circ}\left(x-x_{0}\right)\right) d x=n \kappa_{\Phi} \int_{0}^{R} w(\rho) \rho^{n-1} d \rho,
$$

Moreover, if $\pm w$ is nondecreasing and the composite function $v(x):=w\left(\Phi^{\circ}(x-\right.$ $\left.x_{0}\right)$ ) belongs to $H^{1}\left(B_{R}^{\Phi^{\circ}}\left(x_{0}\right)\right)$, then (see (2.11)

$$
\Phi(\nabla v(x))= \pm w^{\prime}\left(\Phi^{\circ}\left(x-x_{0}\right)\right) \quad \text { for a.e. } x \in B_{R}^{\Phi^{\circ}}\left(x_{0}\right) \text {. }
$$

The geometry of the minimizers of $\mathrm{P}_{\Phi}$ in $\mathbb{R}^{N}$ with prescribed volume $V>0$ is well-known. Indeed, it was established in [12] and [13] (see also [27], 28], [29]) that the minimum of the problem

$$
\min \left\{\mathrm{P}_{\Phi}(E): E \subset \mathbb{R}^{n} \text { with finite perimeter, }|E|=V\right\}
$$

is attained by all balls (2.12) centered at an arbitrary point $x_{0} \in \mathbb{R}^{n}$ and with radius $\rho>0$ chosen so that $\left|B_{\rho}^{\Phi^{\circ}}\left(x_{0}\right)\right|=V$. These balls are called Wulff sets for the $\Phi$-perimeter after the pioneering work of Wulff [30].

Let $\Omega$ be a bounded open set of $\mathbb{R}^{n}$ with Lipschitz boundary, let $m \in \mathbb{R}$ be a constant satisfying (1.3), and let $V_{m}$ be defined by (1.5).

We are interested in the minimum problem

$$
\min \left\{\mathrm{P}_{\Phi}(E): E \text { set of finite perimeter, } E \subset \Omega,|E|=V_{m}\right\} \text {. }
$$

Let $r>0$ be such that $\left|B_{r}^{\Phi^{\circ}}(0)\right|=V_{m}$, that is,

$$
r:=\left(\frac{|\Omega|-m}{2 \kappa_{\Phi}}\right)^{1 / n}
$$

which gives

$$
m=|\Omega|-2 \kappa_{\Phi} r^{n}=|\Omega|-2\left|B_{r}^{\Phi^{\circ}}(0)\right| .
$$

A minimizer of (2.17) is attained at any Wulff set $B_{r}^{\Phi^{\circ}}\left(x_{0}\right)$ contained in $\Omega$, provided there is at least one. For this reason we assume that there exists $y_{0} \in \Omega$ such that

$$
B_{r}^{\Phi^{\circ}}\left(y_{0}\right) \subset \Omega
$$

Our results are strongly hinged on this assumption.

We observe that there exists a Wulff set contained in $\Omega$ provided that $m$ is close to $|\Omega|$, which corresponds to $V_{m}$ and $r$ sufficiently small. 
For technical reasons, related to the proof of the $\Gamma$-limsup inequality, we further assume that, whenever $B_{r}^{\Phi^{\circ}}(x) \subset \Omega$ for $x \in \Omega$, then there exist $y \in \Omega$ and $\delta>0$ with

$$
B_{r}^{\Phi^{\circ}}(x) \subset B_{r+\delta}^{\Phi^{\circ}}(y) \subset \Omega .
$$

Given $\varepsilon>0$, we define

$$
\mathcal{E}_{\varepsilon}(u):=\int_{\Omega}\left(\frac{1}{\varepsilon} W(u(x))+\varepsilon \Phi^{2}(\nabla u(x))\right) d x
$$

for $u \in H^{1}(\Omega)$. Some arguments in what follows will require a localization of this energy, i.e., for every bounded open set $A$ of $\mathbb{R}^{n}$ with Lipschitz boundary and for every $\varepsilon>0$, we define

$$
\mathcal{E}_{\varepsilon}(u, A):=\int_{A}\left(\frac{1}{\varepsilon} W(u(x))+\varepsilon \Phi^{2}(\nabla u(x))\right) d x
$$

if $u \in H^{1}(A)$ and $\mathcal{E}_{\varepsilon}(u, A):=+\infty$ if $u \in L^{1}(A) \backslash H^{1}(A)$. Note that $\mathcal{E}_{\varepsilon}(\cdot, \Omega)=$ $\mathcal{E}_{\varepsilon}(\cdot)$ as defined in (2.22).

Consider the constrained functionals defined on $L^{1}(\Omega)$ by

$$
\mathcal{F}_{\varepsilon}(u):= \begin{cases}\mathcal{E}_{\varepsilon}(u) & \text { if } u \text { satisfies (1.2) } \\ +\infty & \text { otherwise }\end{cases}
$$

and

$$
\mathcal{F}_{0}(u):= \begin{cases}c_{W} \mathrm{P}_{\Phi}(E) & \text { if } u \text { satisfies (1.7), } \\ +\infty & \text { otherwise, }\end{cases}
$$

where $c_{W}$ is defined in (2.7). It is important to observe that in (2.24) the $\Phi$ perimeter $\mathrm{P}_{\Phi}(E)$ is defined by integrating over all the reduced boundary $\partial^{*} E$ of $E$ and not only on $\Omega \cap \partial^{*} E$, i.e., we consider the $\Phi$-perimeter in $\mathbb{R}^{n}$ and not the relative $\Phi$-perimeter in $\Omega$.

A minimizer of $\mathcal{F}_{0}$ is a function of the form $u_{0}=1-2 \chi_{E_{0}}$, where $E_{0} \subset \Omega$ is a minimizer of (2.17). Hence, $E_{0}$ has the form $B_{r}^{\Phi^{\circ}}\left(x_{0}\right)$, with $B_{r}^{\Phi^{\circ}}\left(x_{0}\right) \subset \Omega$ and $r$ defined by (2.18). Then (2.13) gives

$$
\mathrm{P}_{\Phi}\left(E_{0}\right)=\mathrm{P}_{\Phi}\left(B_{r}^{\Phi^{\circ}}\left(x_{0}\right)\right)=n \kappa_{\Phi} r^{n-1} .
$$

We now state the main result of this section.

Theorem 2.1 The family $\left\{\mathcal{F}_{\varepsilon}\right\} \Gamma$-converges in $L^{1}(\Omega)$ to $\mathcal{F}_{0}$.

If the boundary condition $u=1$ on $\partial \Omega$ is omitted and $\mathrm{P}_{\Phi}(E)$ is replaced by by $\mathrm{P}_{\Phi}(E, \Omega):=\int_{\Omega \cap \partial^{*} E} \Phi\left(\nu_{E}\right) d \mathcal{H}^{n-1}$, this result has been established in [20], [21], [26] for the isotropic scalar-valued case, in [14] for the isotropic vectorvalued case, in [6], 23] for the anistropic, scalar-valued case, and in [4] for the anisotropic, vector-valued case (see also [7]). In the proof below we show how to take into account the boundary condition. 
Proof of Theorem 2.1. Similarly to (2.22) and (2.23), we localize (2.24) as

$$
\mathcal{E}_{0}(u, A):=c_{W} \mathrm{P}_{\Phi}(E, A)
$$

if

$$
u=1-2 \chi_{E} \text {, with } E \subset A \text { with finite perimeter in } A \text {, }
$$

where

$$
\mathrm{P}_{\Phi}(E, A):=\int_{A \cap \partial^{*} E} \Phi\left(\nu_{E}(x)\right) d \mathcal{H}^{n-1}(x)
$$

is the the relative $\Phi$-perimeter of $E$ in $A$. We extend $\mathcal{E}_{0}(\cdot, A)$ to $L^{1}(A)$ by setting $\mathcal{E}_{0}(u, A):=+\infty$ if (2.26) is not satisfied. By [6, Theorem 3.5(i)] the family $\left\{\mathcal{E}_{\varepsilon}(\cdot, A)\right\} \Gamma$-converges in $L^{1}(A)$ to $\mathcal{E}_{0}(\cdot, A)$.

Fix a sequence $\varepsilon_{k} \rightarrow 0^{+}$and define

$$
\mathcal{F}_{0}^{\prime}:=\Gamma-\liminf _{k \rightarrow \infty} \mathcal{F}_{\varepsilon_{k}} \quad \text { and } \quad \mathcal{F}_{0}^{\prime \prime}:=\Gamma-\limsup _{k \rightarrow \infty} \mathcal{F}_{\varepsilon_{k}} .
$$

We prove that $\mathcal{F}_{0} \leq \mathcal{F}_{0}^{\prime}$. Let $u \in L^{1}(\Omega)$ be such that $\mathcal{F}_{0}^{\prime}(u)<+\infty$. Then there exists a sequence $\left\{u_{k}\right\}$ converging to $u$ strongly in $L^{1}(\Omega)$ and such that

$$
\liminf _{k \rightarrow \infty} \mathcal{F}_{\varepsilon_{k}}\left(u_{k}\right)=\mathcal{F}_{0}^{\prime}(u)<+\infty .
$$

Passing to a subsequence, not relabeled, we may assume that the liminf is a limit and that $\mathcal{F}_{\varepsilon_{k}}\left(u_{k}\right)<+\infty$ for every $k$. By (1.2) we have that $u_{k} \in H^{1}(\Omega)$, $\int_{\Omega} u_{k}(x) d x=m$, and $u_{k}=1$ on $\partial \Omega$. Fix a bounded open set $A$ of $\mathbb{R}^{n}$ with Lipschitz boundary such that $\bar{\Omega} \subset A$, and extend $u_{k}$ and $u$ to $A$ by setting $u_{k}=u=1$ on $A \backslash \Omega$. Then $u_{k} \in H^{1}(A)$ and $\left\{u_{k}\right\}$ converges to $u$ strongly in $L^{1}(A)$. Hence, by [6. Theorem 3.5(i)],

$$
\mathcal{E}_{0}(u, A) \leq \lim _{k \rightarrow \infty} \mathcal{E}_{\varepsilon_{k}}\left(u_{k}, A\right)<+\infty .
$$

Therefore, there exists a set $E \subset A$ with finite perimeter such that $u=1-2 \chi_{E}$ in $A$. Since $u=1$ in $A \backslash \Omega$, it follows that $E \subset \Omega$ up to a set of measure zero. Hence, $\mathcal{E}_{0}(u, A)=\mathrm{P}_{\Phi}(E, A)=\mathrm{P}_{\Phi}(E)=\mathcal{F}_{0}(u)$. On the other hand, $\mathcal{E}_{\varepsilon_{k}}\left(u_{k}, A\right)=\mathcal{F}_{\varepsilon_{k}}\left(u_{k}\right)$, since $W\left(u_{k}\right)=W(1)=0$ and $\Phi^{2}\left(\nabla u_{k}\right)=\Phi^{2}(0)=0$ in $A \backslash \Omega$. Together with (2.27), this shows that

$$
\mathcal{F}_{0}(u) \leq \liminf _{k \rightarrow \infty} \mathcal{F}_{\varepsilon_{k}}\left(u_{k}\right)=\mathcal{F}_{0}^{\prime}(u) .
$$

This concludes the proof of the inequality $\mathcal{F}_{0} \leq \mathcal{F}_{0}^{\prime}$.

We now prove that $\mathcal{F}_{0}^{\prime \prime} \leq \mathcal{F}_{0}$. Let $u \in L^{1}(\Omega)$ be such that $\mathcal{F}_{0}(u)<+\infty$. By (1.7) there exists a set $E \subset \Omega$ with finite perimeter such that $u=1-2 \chi_{E}$. Since $\Omega$ has a Lipschitz boundary, there exists a sequence of sets $\left\{E_{j}\right\}$ of finite perimeter such that $\chi_{E_{j}} \rightarrow \chi_{E}$ in $L^{1}(\Omega), \mathrm{P}_{\Phi}\left(E_{j}\right) \rightarrow \mathrm{P}_{\Phi}(E), E_{j} \subset \subset \Omega$, and $\left|E_{j}\right|=|E|$ for every $j$. One way to construct $\left\{E_{j}\right\}$ is to consider a sequence $\left\{r_{j}\right\}$ of retractions $r_{j}: \mathbb{R}^{n} \rightarrow \mathbb{R}^{n}$ of class $C^{1}$ such that $\operatorname{supp}\left(r_{j}-\mathrm{id}\right) \subset \subset \mathbb{R}^{n}$, $r_{j}-\mathrm{id} \rightarrow 0$ in $C_{c}^{1}\left(\mathbb{R}^{n} ; \mathbb{R}^{n}\right)$, and $r_{j}(\bar{\Omega}) \subset \Omega$, where id is the identity map (for the 
existence of these retractions see, e.g., [11, Proposition 1.2]). For $j$ large enough $r_{j}$ is invertible and $r_{j}^{-1}-\mathrm{id} \rightarrow 0$ in $C_{c}^{1}\left(\mathbb{R}^{n} ; \mathbb{R}^{n}\right)$. It suffices to take $E_{j}:=r_{j}(E)$.

Let $u_{j}:=1-2 \chi_{E_{j}}$. Since $\mathrm{P}_{\Phi}\left(E_{j}, \Omega\right)=\mathrm{P}_{\Phi}\left(E_{j}\right)$, we have $\mathcal{E}_{0}\left(u_{j}, \Omega\right)=$ $\mathrm{P}_{\Phi}\left(E_{j}\right)$. By [6, Theorem 3.5(ii)] for every $j$ there exists a sequence $\left\{u_{j}^{k}\right\}$ converging to $u_{j}$ in $L^{1}(\Omega)$ such that

$$
\mathrm{P}_{\Phi}\left(E_{j}\right)=\mathcal{E}_{0}\left(u_{j}, \Omega\right)=\limsup _{k \rightarrow \infty} \mathcal{E}_{\varepsilon_{k}}\left(u_{j}^{k}, \Omega\right) \quad \text { and } \quad \int_{\Omega} u_{j}^{k}(x) d x=m .
$$

Since $E_{j} \subset \subset \Omega$, the construction used in [6, Theorem 3.5(ii)] allows us to deduce that $u_{j}^{k} \in H^{1}(\Omega)$ and that it is possible to assume $u_{j}^{k}=1$ on $\partial \Omega$. Hence, $\mathcal{E}_{\varepsilon_{k}}\left(u_{j}^{k}, \Omega\right)=\mathcal{F}_{\varepsilon_{k}}\left(u_{j}^{k}\right)$, so that (2.28) gives

$$
\mathrm{P}_{\Phi}\left(E_{j}\right) \geq \limsup _{k \rightarrow \infty} \mathcal{F}_{\varepsilon_{k}}\left(u_{j}^{k}\right) \geq \mathcal{F}_{0}^{\prime \prime}\left(u_{j}\right)
$$

Letting $j \rightarrow \infty$ and using the lower semicontinuity of $\mathcal{F}_{0}^{\prime \prime}$ and the fact that $\mathrm{P}_{\Phi}\left(E_{j}\right) \rightarrow \mathrm{P}_{\Phi}(E)$, we obtain $\mathcal{F}_{0}(u)=\mathrm{P}_{\Phi}(E) \geq \mathcal{F}_{0}^{\prime \prime}(u)$, which shows that $\mathcal{F}_{0} \geq \mathcal{F}_{0}^{\prime \prime}$.

\section{The Liminf Inequality}

By (2.24) and (2.25), the $\Gamma$-liminf inequality (1.10) is a consequence of the following theorem.

Theorem 3.1 Let $\left\{u_{\varepsilon}\right\}$ be a sequence of functions satisfying (1.2) and converging strongly in $L^{1}(\Omega)$ to a minimizer $u_{0}=1-2 \chi_{E_{0}}$ of $\mathcal{F}_{0}$. Then

$$
\liminf _{\varepsilon \rightarrow 0+} \frac{\mathcal{E}_{\varepsilon}\left(u_{\varepsilon}\right)-n \kappa_{\Phi} c_{W} r^{n-1}}{\varepsilon} \geq 0 .
$$

Proof. We begin by giving an outline of the proof. The first step is to replace $u_{\varepsilon}$ by a minimizer $\tilde{u}_{\varepsilon}$ of an auxiliary energy where we relax the mass constraint in (1.2) with an integral inequality. The advantage in doing this is that we can use a truncation argument to prove that $\tilde{u}_{\varepsilon} \leq 1$ in $\Omega$. This allows us to use a convex symmetrization argument to reduce the energy by replacing $\tilde{u}_{\varepsilon}$ with a "radial" function $\hat{w}_{\varepsilon}$, i.e., a function of the form $\hat{w}_{\varepsilon}(x)=\bar{w}_{\varepsilon}\left(\Phi^{\circ}(x)\right)$ defined on the ball $B_{R}^{\Phi^{\circ}}(0)$ with the same volume as $\Omega$.

To be precise, $\hat{w}_{\varepsilon}$ is defined as a "radial" minimizer of a problem in $B_{R}^{\Phi^{\circ}}(0)$ with a suitable inequality constraint on the mass. The one-dimensional function $\bar{w}_{\varepsilon}$ satisfies an Euler-Lagrange equation with a Lagrange multiplier $\lambda_{\varepsilon}$ such that $\varepsilon \lambda_{\varepsilon} \rightarrow 0$ as $\varepsilon \rightarrow 0+$. The choice of the inequality constraint allows us to prove that $\lambda_{\varepsilon} \geq 0$, which will be important in what follows.

To estimate the energy of $\bar{w}_{\varepsilon}$ it is convenient to consider the change of variables $\rho=r+\varepsilon t$, where $r$ is defined in (2.18), and to introduce the function $w_{\varepsilon}(t):=\bar{w}_{\varepsilon}(r+\varepsilon t)$ for $-\frac{r}{\varepsilon} \leq t \leq \frac{R-r}{\varepsilon}$. Now the context of our problem has 
been reduced to a simpler one-dimensional setting. Indeed, it turns out that to prove (3.1) it is enough to show that

$$
\liminf _{\varepsilon \rightarrow 0+} \frac{\mathcal{H}_{\varepsilon}\left(w_{\varepsilon}\right)-c_{W} r^{n-1}}{\varepsilon} \geq 0
$$

where the functional

$$
\mathcal{H}_{\varepsilon}(w):=\int_{-\frac{r}{\varepsilon}}^{\frac{R-r}{\varepsilon}}\left(W(w(t))+\left|w^{\prime}(t)\right|^{2}\right)(r+\varepsilon t)^{n-1} d t
$$

does not contain singular terms in $\varepsilon$.

The proof of (3.2) is based on several delicate estimates on $w_{\varepsilon}$. We first show that $w_{\varepsilon}$ vanishes at a point $\delta_{\varepsilon}$, with $\varepsilon \delta_{\varepsilon} \rightarrow 0$ as $\varepsilon \rightarrow 0+$, and introduce the shifted function $\check{w}_{\varepsilon}(t):=w_{\varepsilon}\left(t+\delta_{\varepsilon}\right)$. Then we prove that $\check{w}_{\varepsilon} \rightarrow z$ in $H_{\text {loc }}^{1}$, where $z$ is the "optimal profile" introduced in (2.5), and that $\lambda_{\varepsilon} \rightarrow(n-1) c_{W}$ as $\varepsilon \rightarrow 0+$. Next we derive some technical estimates on $\check{w}_{\varepsilon}$ using arguments from the theory of ordinary differential equations, which rely on the fact that $\check{w}_{\varepsilon}(0)=0$. These estimates allow us to show that $\liminf _{\varepsilon} \delta_{\varepsilon} \geq 0$ and to finally prove (3.2).

We divide the proof into a series of steps.

Step 1. Here we replace $u_{\varepsilon}$ by a minimizer $\tilde{u}_{\varepsilon}$ of an auxiliary energy where we relax the mass constraint in (1.2) with an integral inequality.

To be precise, we introduce the functional $\tilde{\mathcal{F}}_{\varepsilon}$ defined by

$$
\tilde{\mathcal{F}}_{\varepsilon}(u):=\mathcal{E}_{\varepsilon}(u)
$$

if

$$
u \in H^{1}(\Omega), \quad \int_{\Omega} u(x) d x \leq m, \quad \text { and } \quad u=1 \text { on } \partial \Omega .
$$

We extend $\tilde{\mathcal{F}}_{\varepsilon}$ to $L^{1}(\Omega)$ by setting $\tilde{\mathcal{F}}_{\varepsilon}(u):=+\infty$ if (3.4) is not satisfied. Then, reasoning as in Theorem 2.1, we can show that the $\Gamma$-limit of $\left\{\tilde{\mathcal{F}}_{\varepsilon}\right\}$ is the functional $\tilde{\mathcal{F}}_{0}$ defined by

$$
\tilde{\mathcal{F}}_{0}(u):=c_{W} \mathrm{P}_{\Phi}(E)
$$

if

$$
u=1-2 \chi_{E}, \quad E \subset \Omega, \quad \mathrm{P}(E)<+\infty, \text { and } \int_{\Omega} u(x) d x \leq m,
$$

while $\tilde{\mathcal{F}}_{0}(u):=+\infty$ if (3.5) is not satisfied.

Let $\tilde{u}_{\varepsilon}$ be a minimizer of $\tilde{\mathcal{F}}_{\varepsilon}$, whose existence can be justified by the Direct Method of the Calculus of Variations. Then

$$
\tilde{\mathcal{F}}_{\varepsilon}\left(\tilde{u}_{\varepsilon}\right) \leq \mathcal{E}_{\varepsilon}\left(u_{\varepsilon}\right) .
$$

Note that, by standard properties of $\Gamma$-convergence, we have that the sequence

$$
\left\{\tilde{\mathcal{F}}_{\varepsilon}\left(\tilde{u}_{\varepsilon}\right)\right\} \text { is bounded }
$$


and $\left\{\tilde{u}_{\varepsilon}\right\}$ converges strongly in $L^{1}(\Omega)$ to $u_{0}=1-2 \chi_{E_{0}}$, where $E_{0}$ satisfies $\mathrm{P}_{\Phi}\left(E_{0}\right) \leq \mathrm{P}_{\Phi}(E)$ for every set $E \subset \Omega$ with finite perimeter and such that

$$
\frac{|\Omega|-m}{2} \leq|E|
$$

We claim that

$$
\frac{|\Omega|-m}{2}=\left|E_{0}\right| .
$$

Since the ball $B_{r}^{\Phi^{\circ}}\left(y_{0}\right)$ introduced in (2.20) is contained in $\Omega$ and satisfies $\frac{|\Omega|-m}{2}=\left|B_{r}^{\Phi^{\circ}}\left(y_{0}\right)\right|$, we have

$$
\mathrm{P}_{\Phi}\left(E_{0}\right) \leq \mathrm{P}_{\Phi}\left(B_{r}^{\Phi^{\circ}}\left(y_{0}\right)\right) .
$$

Let $\rho \geq r$ be such that $\left|E_{0}\right|=\left|B_{\rho}^{\Phi^{\circ}}\left(y_{0}\right)\right|$. Then for every $F \subset \mathbb{R}^{n}$ with finite perimeter and with $|F|=\left|E_{0}\right|$, by the minimality of the Wulff shape in $\mathbb{R}^{n}$ (see [12]) it follows

$$
\mathrm{P}_{\Phi}\left(B_{\rho}^{\Phi^{\circ}}\left(y_{0}\right)\right) \leq \mathrm{P}_{\Phi}(F) .
$$

From (2.13), (3.9), and (3.10) we obtain $\mathrm{P}_{\Phi}\left(E_{0}\right) \leq \mathrm{P}_{\Phi}(F)$ for every $F \subset \mathbb{R}^{n}$ with finite perimeter and with $|F|=\left|E_{0}\right|$. Since the Wulff sets are the unique minimizers of $\mathrm{P}_{\Phi}$ in $\mathbb{R}^{n}$ under the volume constraint (see [13]), there exists $x_{0} \in \Omega$ such that $E_{0}=B_{\rho}^{\Phi^{\circ}}\left(x_{0}\right)$. By (2.13) and (3.9) it follows that $\rho=r$ and that (3.8) holds.

Next we prove that $\tilde{u}_{\varepsilon} \leq 1$ a.e. in $\Omega$. Let $u_{1}:=\min \left\{\tilde{u}_{\varepsilon}, 1\right\}$. Assume, by contradiction, that $\left|\left\{\tilde{u}_{\varepsilon}>1\right\}\right|>0$. Since $W(1)=0$ and $W(s)>0$ for $s>1$ by (2.2) and (2.4), we have that $W\left(u_{1}(x)\right) \leq W\left(\tilde{u}_{\varepsilon}(x)\right)$ for a.e. $x \in \Omega$, and the inequality is strict for a.e. $x \in\left\{\tilde{u}_{\varepsilon}>1\right\}$. This implies that

$$
\int_{\Omega} W\left(u_{1}(x)\right) d x<\int_{\Omega} W\left(\tilde{u}_{\varepsilon}(x)\right) d x .
$$

Since $\nabla u_{1}=\nabla \tilde{u}_{\varepsilon}$ a.e. on $\left\{\tilde{u}_{\varepsilon} \leq 1\right\}$ and $\nabla u_{1}=0$ a.e. on $\left\{\tilde{u}_{\varepsilon}>1\right\}$, we have also $\Phi\left(\nabla u_{1}(x)\right) \leq \Phi\left(\nabla \tilde{u}_{\varepsilon}(x)\right)$ for a.e. $x \in \Omega$, which implies

$$
\int_{\Omega} \Phi^{2}\left(\nabla u_{1}(x)\right) d x \leq \int_{\Omega} \Phi^{2}\left(\nabla \tilde{u}_{\varepsilon}(x)\right) d x .
$$

Noting that $u_{1}$ satisfies (3.4), the previous inequalities give $\tilde{\mathcal{F}}_{\varepsilon}\left(u_{1}\right)<\tilde{\mathcal{F}}_{\varepsilon}\left(\tilde{u}_{\varepsilon}\right)$, which contradicts the minimality of $\tilde{u}_{\varepsilon}$. This proves that $\tilde{u}_{\varepsilon} \leq 1$ a.e. in $\Omega$.

Step 2. In this step we use "convex" rearrangements to replace $\tilde{u}_{\varepsilon}$ by a "radial" function $\hat{w}_{\varepsilon}$, i.e., a function depending on $x$ only through $\Phi^{\circ}(x)$.

Define $\tilde{v}_{\varepsilon}:=1-\tilde{u}_{\varepsilon} \in H_{0}^{1}(\Omega)$ and observe that

$$
\mathcal{E}_{\varepsilon}\left(\tilde{u}_{\varepsilon}\right)=\int_{\Omega}\left(\frac{1}{\varepsilon} W\left(1-\tilde{v}_{\varepsilon}(x)\right)+\varepsilon \Phi^{2}\left(\nabla \tilde{v}_{\varepsilon}(x)\right)\right) d x .
$$


Since $\tilde{v}_{\varepsilon} \geq 0$, we define the "convex" rearrangement $v_{\varepsilon}^{\star}$ of $\tilde{v}_{\varepsilon}$ as the unique function of the form

$$
v_{\varepsilon}^{\star}(x)=\bar{v}_{\varepsilon}\left(\Phi^{\circ}(x)\right),
$$

with $\bar{v}_{\varepsilon}: \mathbb{R}^{+} \rightarrow \mathbb{R}^{+}$nonincreasing and continuous from the right, and such that $\left|\left\{\tilde{v}_{\varepsilon}>t\right\}\right|=\left|\left\{v_{\varepsilon}^{\star}>t\right\}\right|$ for every $t>0$. It can be shown that

$$
\int_{B_{R}^{\Phi^{\circ}}(0)} W\left(1-v_{\varepsilon}^{\star}(x)\right) d x=\int_{\Omega} W\left(1-\tilde{v}_{\varepsilon}(x)\right) d x,
$$

where $\left|B_{R}^{\Phi^{\circ}}(0)\right|=|\Omega|$. The Pólya-Szegö principle, which holds also for "convex" rearrangements (see [1, Theorem 3.1]), gives $v_{\varepsilon}^{\star} \in H_{0}^{1}\left(B_{R}^{\Phi^{\circ}}(0)\right)$ and

$$
\int_{B_{R}^{\Phi \circ}(0)} \Phi^{2}\left(\nabla v_{\varepsilon}^{\star}(x)\right) d x \leq \int_{\Omega} \Phi^{2}\left(\nabla \tilde{v}_{\varepsilon}(x)\right) d x .
$$

Therefore, we deduce that

$$
\mathcal{E}_{\varepsilon}\left(1-v_{\varepsilon}^{\star}, B_{R}^{\Phi^{\circ}}(0)\right) \leq \tilde{\mathcal{F}}_{\varepsilon}\left(\tilde{u}_{\varepsilon}\right) .
$$

Let $\hat{w}_{\varepsilon}$ be a minimizer of $\mathcal{E}_{\varepsilon}\left(\cdot, B_{R}^{\Phi^{\circ}}(0)\right)$ among all functions $u \in H^{1}\left(B_{R}^{\Phi^{\circ}}(0)\right)$ satisfying

$$
\int_{B_{R}^{\Phi^{\circ}}(0)} u(x) d x \leq m \text { and } u=1 \text { on } \partial B_{R}^{\Phi^{\circ}}(0) .
$$

By (3.12) we have

$$
\mathcal{E}_{\varepsilon}\left(\hat{w}_{\varepsilon}, B_{R}^{\Phi^{\circ}}(0)\right) \leq \mathcal{E}_{\varepsilon}\left(1-v_{\varepsilon}^{\star}, B_{R}^{\Phi^{\circ}}(0)\right) \leq \tilde{\mathcal{F}}_{\varepsilon}\left(\tilde{u}_{\varepsilon}\right) .
$$

Reasoning as in Step 1 , with $\tilde{\mathcal{F}}_{\varepsilon}$ replaced by $\mathcal{E}_{\varepsilon}\left(\cdot, B_{R}^{\Phi^{\circ}}(0)\right)$, we may assume that

$$
\hat{w}_{\varepsilon} \rightarrow 1-2 \chi_{B_{r}^{\Phi^{\circ}}(0)} \text { in } L^{1}\left(B_{R}^{\Phi^{\circ}}(0)\right),
$$

where $r$ is given by (2.18).

Using a symmetrization argument similar to the one above (see (3.11) and (3.12)), we can assume that there exists a function $\bar{w}_{\varepsilon}: \mathbb{R}^{+} \rightarrow \mathbb{R}^{+}$, nondecreasing and continuous from the right, such that

$$
\hat{w}_{\varepsilon}(x)=\bar{w}_{\varepsilon}\left(\Phi^{\circ}(x)\right) .
$$

By (2.16) and (3.11) we have $\Phi\left(\nabla \hat{w}_{\varepsilon}(x)\right)=\bar{w}_{\varepsilon}^{\prime}\left(\Phi^{\circ}(x)\right)$ for a.e. $x \in B_{R}^{\Phi^{\circ}}(0)$, and so (2.15) yields

$$
\mathcal{E}_{\varepsilon}\left(\hat{w}_{\varepsilon}, B_{R}^{\Phi^{\circ}}(0)\right)=n \kappa_{\Phi} \int_{0}^{R}\left(\frac{1}{\varepsilon} W\left(\bar{w}_{\varepsilon}(\rho)\right)+\varepsilon\left|\bar{w}_{\varepsilon}^{\prime}(\rho)\right|^{2}\right) \rho^{n-1} d \rho .
$$

Step 3. In this step we prove some elementary properties of the one-dimensional function $\bar{w}_{\varepsilon}$ introduced in (3.15). 
By the minimality of $\hat{w}_{\varepsilon}$, restricting our attention to functions of the form $w \circ \Phi^{\circ}$ and using (2.15) and (2.16), we deduce that $\bar{w}_{\varepsilon}$ is a minimizer of

$$
\mathcal{G}_{\varepsilon}(w):=\int_{0}^{R}\left(\frac{1}{\varepsilon} W(w(\rho))+\varepsilon\left|w^{\prime}(\rho)\right|^{2}\right) \rho^{n-1} d \rho
$$

among all functions $w \in H_{\mathrm{loc}}^{1}(0, R)$ subject to the constraints

$$
\int_{0}^{R}\left|w^{\prime}(\rho)\right|^{2} \rho^{n-1} d \rho<+\infty, \quad w(R)=1, \quad n \kappa_{\Phi} \int_{0}^{R} w(\rho) \rho^{n-1} d \rho \leq m .
$$

Note that if $w$ satisfies the first two conditions in (3.18) then

$$
|w(\rho)| \leq 1+\int_{\rho}^{R}\left|w^{\prime}(\sigma)\right| d \sigma
$$

hence

$$
\left|w(\rho) \rho^{n-1}\right| \leq \rho^{n-1}+\int_{\rho}^{R}\left|w^{\prime}(\sigma)\right| \sigma^{n-1} d \sigma .
$$

By Hölder's inequality we obtain

$$
\left|w(\rho) \rho^{n-1}\right| \leq R^{n-1}+\left(\frac{R^{n}}{n}\right)^{1 / 2}\left(\int_{0}^{R}\left|w^{\prime}(\sigma)\right|^{2} \sigma^{n-1} d \sigma\right)^{1 / 2}
$$

hence

$$
w(\rho) \rho^{n-1} \quad \text { is bounded in }(0, R) .
$$

This implies that the integral in the last inequality in (3.18) is well defined.

Reasoning by truncation as at the end of Step 1, we can prove that

$$
\bar{w}_{\varepsilon}(\rho) \leq 1 \quad \text { for } 0<\rho<R .
$$

Using the equalities $\hat{w}_{\varepsilon}=\bar{w}_{\varepsilon} \circ \Phi^{\circ}$ and $\chi_{B_{r}^{\Phi \circ}(0)}=\chi_{[0, r)} \circ \Phi^{\circ}$, by (3.14) we obtain that

$$
\bar{w}_{\varepsilon} \rightarrow 1-2 \chi_{[0, r)} \text { in } L^{1}(0, R) .
$$

Since each $\bar{w}_{\varepsilon}$ is nondecreasing, it follows that there is pointwise convergence at every $\rho$, with the possible exceptions of 0 and $r$. In particular, we have

$$
\bar{w}_{\varepsilon}(\rho) \rightarrow\left\{\begin{aligned}
-1 & \text { if } 0<\rho<r, \\
1 & \text { if } r<\rho \leq R,
\end{aligned}\right.
$$

where the case $\rho=R$ can be obtained by (3.20). In turn, for every $0<\rho_{0}<R$ the sequence

$$
\left\{\bar{w}_{\varepsilon}\right\} \quad \text { is bounded in } L^{\infty}\left(\left[\rho_{0}, R\right]\right) .
$$

Step 4. Here we derive the Euler-Lagrange equation for $\bar{w}_{\varepsilon}$, and we prove that the corresponding Lagrange multipliers $\lambda_{\varepsilon}$ satisfy

$$
\lim _{\varepsilon \rightarrow 0+} \varepsilon \lambda_{\varepsilon}=0 .
$$


We claim that $\bar{w}_{\varepsilon} \in C^{2}(0, R)$ and satisfies the Euler-Lagrange equation

$$
-2 \varepsilon \bar{w}_{\varepsilon}^{\prime \prime}(\rho)-\frac{2(n-1) \varepsilon}{\rho} \bar{w}_{\varepsilon}^{\prime}(\rho)+\frac{1}{\varepsilon} W^{\prime}\left(\bar{w}_{\varepsilon}(\rho)\right)=-\lambda_{\varepsilon} \leq 0
$$

for some constant $\lambda_{\varepsilon} \geq 0$. To see this, let $\varphi \in C_{c}^{\infty}(0, R)$ be nonnegative. For $t>0$ the function $\bar{w}_{\varepsilon}-t \varphi$ fulfills (3.18), and so

$$
\mathcal{G}_{\varepsilon}\left(\bar{w}_{\varepsilon}\right) \leq \mathcal{G}_{\varepsilon}\left(\bar{w}_{\varepsilon}-t \varphi\right) .
$$

Thus the derivative of the function $t \mapsto \mathcal{G}_{\varepsilon}\left(\bar{w}_{\varepsilon}-t \varphi\right)$ is greater than or equal to 0 at $t=0$. This gives

$$
\int_{0}^{R}\left(\frac{1}{\varepsilon} W^{\prime}\left(\bar{w}_{\varepsilon}(\rho)\right) \varphi(\rho)+2 \varepsilon \bar{w}_{\varepsilon}^{\prime}(\rho) \varphi^{\prime}(\rho)\right) \rho^{n-1} d \rho \leq 0
$$

for all nonnegative $\varphi \in C_{c}^{\infty}(0, R)$, which shows that $-2 \varepsilon\left(\bar{w}_{\varepsilon}^{\prime}(\rho) \rho^{n-1}\right)^{\prime}+$ $\frac{1}{\varepsilon} W^{\prime}\left(\bar{w}_{\varepsilon}(\rho)\right) \rho^{n-1}$ is nonpositive in the sense of distributions.

On the other hand, if we consider $\psi \in C_{c}^{\infty}(0, R)$ such that $\int_{0}^{R} \psi(\rho) \rho^{n-1} d \rho=$ 0 , then $\bar{w}_{\varepsilon}+t \psi$ satisfies (3.18) for all $t \in \mathbb{R}$, and so

$$
\int_{0}^{R}\left(\frac{1}{\varepsilon} W^{\prime}\left(\bar{w}_{\varepsilon}(\rho)\right) \psi(\rho)+2 \varepsilon \bar{w}_{\varepsilon}^{\prime}(\rho) \psi^{\prime}(\rho)\right) \rho^{n-1} d \rho=0
$$

for all $\psi \in C_{c}^{\infty}(0, R)$ with $\int_{0}^{R} \psi(\rho) \rho^{n-1} d \rho=0$. We now use a classical argument (see, e.g., [17, Lemma 7.3]) to show that (3.26) implies that there exists a constant $\lambda_{\varepsilon}$ such that

$$
-2 \varepsilon\left(\bar{w}_{\varepsilon}^{\prime}(\rho) \rho^{n-1}\right)^{\prime}+\frac{1}{\varepsilon} W^{\prime}\left(\bar{w}_{\varepsilon}(\rho)\right) \rho^{n-1}=-\lambda_{\varepsilon} \rho^{n-1}
$$

in the sense of distributions in $(0, R)$.

Fix $\varphi_{1} \in C_{c}^{\infty}(0, R)$ with $\int_{0}^{R} \varphi_{1}(\rho) \rho^{n-1} d \rho=1$. Given $\varphi \in C_{c}^{\infty}(0, R)$, we can write $\varphi=c_{\varphi} \varphi_{1}+\psi$, where $c_{\varphi}:=\int_{0}^{R} \varphi(\rho) \rho^{n-1} d \rho$ and $\psi \in C_{c}^{\infty}(0, R)$ satisfies $\int_{0}^{R} \psi(\rho) \rho^{n-1} d \rho=0$. Hence, using (3.26), we obtain

$$
\int_{0}^{R}\left(\frac{1}{\varepsilon} W^{\prime}\left(\bar{w}_{\varepsilon}(\rho)\right) \varphi(\rho)+2 \varepsilon \bar{w}_{\varepsilon}^{\prime}(\rho) \varphi^{\prime}(\rho)\right) \rho^{n-1} d \rho=-\lambda_{\varepsilon} \int_{0}^{R} \varphi(\rho) \rho^{n-1} d \rho,
$$

where

$$
\lambda_{\varepsilon}:=-\int_{0}^{R}\left(\frac{1}{\varepsilon} W^{\prime}\left(\bar{w}_{\varepsilon}(\rho)\right) \varphi_{1}(\rho)+2 \varepsilon \bar{w}_{\varepsilon}^{\prime}(\rho) \varphi_{1}^{\prime}(\rho)\right) \rho^{n-1} d \rho .
$$

This concludes the proof of (3.27).

By (3.25) it follows that $\lambda_{\varepsilon} \geq 0$. Using the facts that $W$ is of class $C^{1}$ and that $\bar{w}_{\varepsilon}$ is bounded on $\left[\rho_{0}, R\right]$ for every $0<\rho_{0}<R$ by (3.19), we deduce that $\bar{w}_{\varepsilon} \in C^{2}(0, R)$ and that (3.24) and (3.27) are satisfied pointwise. 
Next we prove (3.23). By (3.28),

$$
\varepsilon \lambda_{\varepsilon}=-\int_{0}^{R} W^{\prime}\left(\bar{w}_{\varepsilon}(\rho)\right) \varphi_{1}(\rho) \rho^{n-1} d \rho-\int_{0}^{R} 2 \varepsilon^{2} \bar{w}_{\varepsilon}^{\prime}(\rho) \varphi_{1}^{\prime}(\rho) \rho^{n-1} d \rho .
$$

Since $\varphi_{1}$ has compact support in $(0, R)$, the first integral tends to zero in view of (2.1), (2.4), (3.21), and (3.22), while the second integral goes to zero since the sequence $\left\{\varepsilon\left|\bar{w}_{\varepsilon}^{\prime}\right|^{2} \rho^{n-1}\right\}$ is bounded in $L^{1}(0, R)$ by (3.7), (3.13), and (3.16). This concludes the proof of (3.23).

Step 5. Here we prove that for $\varepsilon>0$ small enough

$$
\inf \bar{w}_{\varepsilon} \geq-1-\left(\frac{\varepsilon \lambda_{\varepsilon}}{\beta}\right)^{1 /(\beta-1)},
$$

where $1<\beta<2$ is the constant in (2.4).

Integrating (3.27) gives

$$
\begin{aligned}
& \bar{w}_{\varepsilon}^{\prime}\left(\rho_{2}\right) \rho_{2}^{n-1}-\bar{w}_{\varepsilon}^{\prime}\left(\rho_{1}\right) \rho_{1}^{n-1} \\
& =\frac{1}{2 \varepsilon} \int_{\rho_{1}}^{\rho_{2}}\left(\frac{1}{\varepsilon} W^{\prime}\left(\bar{w}_{\varepsilon}(\rho)\right) \rho^{n-1}+\lambda_{\varepsilon} \rho^{n-1}\right) d \rho
\end{aligned}
$$

for $0<\rho_{1} \leq \rho_{2} \leq R$. Since $\bar{w}_{\varepsilon}(\rho) \leq 1$ for every $0<\rho \leq R$ by (3.20), and $W^{\prime}(s)<0$ for $s<-1$ by (2.1) and (2.2), the integral

$$
\int_{0}^{R}\left(\frac{1}{\varepsilon} W^{\prime}\left(\bar{w}_{\varepsilon}(\rho)\right) \rho^{n-1}+\lambda_{\varepsilon} \rho^{n-1}\right) d \rho
$$

is well-defined as an element of $\mathbb{R} \cup\{-\infty\}$. We claim that

$$
\lim _{\rho \rightarrow 0+} \bar{w}_{\varepsilon}^{\prime}(\rho) \rho^{n-1}=0 .
$$

First, we observe that the limit exists in $\mathbb{R} \cup\{+\infty\}$ by (3.30). If it were different from zero, then there would exist $c_{0}>0$ and $\rho_{0}>0$ such that $\left|\bar{w}_{\varepsilon}^{\prime}(\rho)\right| \geq c_{0} / \rho^{n-1}$ for $0<\rho<\rho_{0}$. It would follow that

$$
\int_{0}^{R}\left|w^{\prime}(\rho)\right|^{2} \rho^{n-1} d \rho \geq c_{0}^{2} \int_{0}^{\rho_{0}} \frac{d \rho}{\rho^{n-1}}=+\infty,
$$

which would contradict the first inequality in (3.18) since $n \geq 2$. This gives (3.31).

To prove (3.29) we first show that

$$
\liminf _{\varepsilon \rightarrow 0+} \inf \bar{w}_{\varepsilon} \geq-1 .
$$

It is enough to prove an estimate from below for those $\varepsilon$ such that inf $\bar{w}_{\varepsilon}<-1$. We claim that for those $\varepsilon$,

$$
\limsup _{\rho \rightarrow 0+}\left(W^{\prime}\left(\bar{w}_{\varepsilon}(\rho)\right)+\varepsilon \lambda_{\varepsilon}\right) \geq 0 .
$$


If not, (3.30) and (3.31) imply that $\bar{w}_{\varepsilon}^{\prime}(\rho) \rho^{n-1}<0$ for $\rho>0$ small enough, and this violates the fact that $\bar{w}_{\varepsilon}$ is nondecreasing. This proves (3.33), which implies that, for inf $\bar{w}_{\varepsilon}<-1$, we have

$$
W^{\prime}\left(\inf \bar{w}_{\varepsilon}\right)+\varepsilon \lambda_{\varepsilon} \geq 0,
$$

where $W^{\prime}(-\infty)$ denotes the limsup of $W^{\prime}(s)$ as $s \rightarrow-\infty$.

Since $W^{\prime}(s)<0$ for every $-\infty \leq s<-1$, by (2.1), (2.2), and (2.3), inequality (3.32) follows from (3.23).

By (2.1), (2.4), (3.32), and (3.34), if $\varepsilon>0$ is small enough and inf $\bar{w}_{\varepsilon}<-1$ we have

$$
\beta\left(-\inf \bar{w}_{\varepsilon}-1\right)^{\beta-1} \leq \varepsilon \lambda_{\varepsilon} .
$$

This proves (3.29).

Step 6. In this step we consider the change of variables $\rho=r+\varepsilon t$, we define $w_{\varepsilon}(t):=\bar{w}_{\varepsilon}(r+\varepsilon t)$ for $-\frac{r}{\varepsilon} \leq t \leq \frac{R-r}{\varepsilon}$, and we derive the one-dimensional formulation (3.2) of the problem in terms of the new energy $\mathcal{H}_{\varepsilon}$ introduced in (3.3).

Note that $w_{\varepsilon}\left(\frac{R-r}{\varepsilon}\right)=1$ and $w_{\varepsilon}$ is nondecreasing. By (3.29), for $\varepsilon>0$ small enough we have

$$
\inf w_{\varepsilon} \geq-1-\left(\frac{\varepsilon \lambda_{\varepsilon}}{\beta}\right)^{1 /(\beta-1)} .
$$

In particular, using also (3.20) and (3.23), for all $\varepsilon>0$ sufficiently small we get

$$
-2 \leq w_{\varepsilon}(t) \leq 1
$$

for all $-\frac{r}{\varepsilon} \leq t \leq \frac{R-r}{\varepsilon}$.

Moreover, by (13.24),$w_{\varepsilon}$ satisfies the Euler-Lagrange equation

$$
-2 w_{\varepsilon}^{\prime \prime}(t)-2(n-1) \varepsilon \frac{w_{\varepsilon}^{\prime}(t)}{r+\varepsilon t}+W^{\prime}\left(w_{\varepsilon}(t)\right)=-\varepsilon \lambda_{\varepsilon} \leq 0,
$$

and by (3.18),

$$
\int_{-\frac{r}{\varepsilon}}^{\frac{R-r}{\varepsilon}} w_{\varepsilon}(t)(r+\varepsilon t)^{n-1} d t \leq \frac{m}{n \kappa_{\Phi} \varepsilon} .
$$

Observe that, setting

$$
w_{0}(t):=\left\{\begin{aligned}
-1 & \text { if } t<0 \\
1 & \text { if } t>0
\end{aligned}\right.
$$

we have

$$
\begin{gathered}
\int_{-\frac{r}{\varepsilon}}^{\frac{R-r}{\varepsilon}} w_{0}(t)(r+\varepsilon t)^{n-1} d t=\int_{0}^{\frac{R-r}{\varepsilon}}(r+\varepsilon t)^{n-1} d t-\int_{-\frac{r}{\varepsilon}}^{0}(r+\varepsilon t)^{n-1} d t \\
=\frac{1}{n \varepsilon}\left[(r+\varepsilon t)^{n}\right]_{0}^{\frac{R-r}{\varepsilon}}-\frac{1}{n \varepsilon}\left[(r+\varepsilon t)^{n}\right]_{-\frac{r}{\varepsilon}}^{0}=\frac{1}{n \varepsilon}\left(R^{n}-2 r^{n}\right)=\frac{m}{n \kappa_{\Phi} \varepsilon}
\end{gathered}
$$


where the last equality follows from (2.19), taking into account the fact that $\kappa_{\Phi} R^{n}=\left|B_{R}^{\Phi^{\circ}}(0)\right|=|\Omega|$. Thus, (3.37) is equivalent to

$$
\int_{-\frac{r}{\varepsilon}}^{\frac{R-r}{\varepsilon}}\left(w_{\varepsilon}(t)-w_{0}(t)\right)(r+\varepsilon t)^{n-1} d t \leq 0 .
$$

The minimality of $\bar{w}_{\varepsilon}$ for $\mathcal{G}_{\varepsilon}$ and a change of variables show that $w_{\varepsilon}$ is a minimizer of the functional $\mathcal{H}_{\varepsilon}$ defined in (3.3) over all $w \in H_{\text {loc }}^{1}\left(-\frac{r}{\varepsilon}, \frac{R-r}{\varepsilon}\right) \cap$ $H^{1}\left(0, \frac{R-r}{\varepsilon}\right)$ satisfying $w\left(\frac{R-r}{\varepsilon}\right)=1$ and

$$
\int_{-\frac{r}{\varepsilon}}^{\frac{R-r}{\varepsilon}}\left(w(t)-w_{0}(t)\right)(r+\varepsilon t)^{n-1} d t \leq 0 .
$$

By (3.6), (3.12), (3.13), (3.16), and (3.17) we have

$$
\mathcal{H}_{\varepsilon}\left(w_{\varepsilon}\right)=\mathcal{G}_{\varepsilon}\left(\bar{w}_{\varepsilon}\right)=\frac{1}{n \kappa_{\Phi}} \mathcal{E}_{\varepsilon}\left(\hat{w}_{\varepsilon}, B_{R}^{\Phi^{\circ}}(0)\right) \leq \frac{1}{n \kappa_{\Phi}} \tilde{\mathcal{F}}_{\varepsilon}\left(\tilde{u}_{\varepsilon}\right) \leq \frac{1}{n \kappa_{\Phi}} \mathcal{E}_{\varepsilon}\left(u_{\varepsilon}\right) .
$$

Therefore, in order to prove (3.1) it is enough to show that (3.2) holds.

Step 7. Here we prove that the function $w_{\varepsilon}$ obtained in the previous step vanishes at a point $\delta_{\varepsilon}$, and that

$$
\lim _{\varepsilon \rightarrow 0^{+}} \varepsilon \delta_{\varepsilon}=0 .
$$

Let $z$ be the function defined by (2.5). We claim that $w=z$ satisfies (3.40) for all $\varepsilon>0$ sufficiently small. Since $z(t)=w_{0}(t)$ for $|t| \geq \tau_{W}$, (3.40) reduces to

$$
\int_{-\tau_{W}}^{\tau_{W}}\left(z(t)-w_{0}(t)\right)(r+\varepsilon t)^{n-1} d t \leq 0
$$

for all $\varepsilon>0$ sufficiently small, or, equivalently,

$$
\int_{0}^{\tau_{W}}\left(z(t)-w_{0}(t)\right)(r+\varepsilon t)^{n-1} d t \leq-\int_{-\tau_{W}}^{0}\left(z(t)-w_{0}(t)\right)(r+\varepsilon t)^{n-1} d t .
$$

Using the fact that $z-w_{0}$ is odd, a change of variables on the right-hand side leads to

$$
\int_{0}^{\tau_{W}}\left(z(t)-w_{0}(t)\right)(r+\varepsilon t)^{n-1} d t \leq \int_{0}^{\tau_{W}}\left(z(t)-w_{0}(t)\right)(r-\varepsilon t)^{n-1} d t,
$$

which follows from the fact that $z(t)-w_{0}(t) \leq 0$ for all $0 \leq t \leq \tau_{W}$. Hence, $z$ satisfies (3.40) for all $\varepsilon>0$ sufficiently small and so, by the minimality of $w_{\varepsilon}$, we have $\mathcal{H}_{\varepsilon}\left(w_{\varepsilon}\right) \leq \mathcal{H}_{\varepsilon}(z)$.

Moreover, by (2.7),

$$
\begin{aligned}
\mathcal{H}_{\varepsilon}(z) & =\int_{-\tau_{W}}^{\tau_{W}}\left(W(z(t))+\left|z^{\prime}(t)\right|^{2}\right)(r+\varepsilon t)^{n-1} d t \\
& =\int_{-\tau_{W}}^{\tau_{W}}\left(W(z(t))+\left|z^{\prime}(t)\right|^{2}\right) r^{n-1} d t+O\left(\varepsilon^{2}\right)=c_{W} r^{n-1}+O\left(\varepsilon^{2}\right),
\end{aligned}
$$


where we have used the fact

$$
\int_{-\tau_{W}}^{\tau_{W}}\left(W(z(t))+\left|z^{\prime}(t)\right|^{2}\right) t d t=0
$$

since $W$ is even and $z$ is odd. It follows that

$$
\mathcal{H}_{\varepsilon}\left(w_{\varepsilon}\right) \leq \mathcal{H}_{\varepsilon}(z)=c_{W} r^{n-1}+O\left(\varepsilon^{2}\right) .
$$

Fix $0<r_{1}<r<r_{2}<R$. Then $\bar{w}_{\varepsilon}\left(r_{1}\right) \rightarrow-1$ and $\bar{w}_{\varepsilon}\left(r_{2}\right) \rightarrow 1$ by (3.21), and so $w_{\varepsilon}\left(\frac{r_{1}-r}{\varepsilon}\right) \rightarrow-1$ and $w_{\varepsilon}\left(\frac{r_{2}-r}{\varepsilon}\right) \rightarrow 1$ as $\varepsilon \rightarrow 0^{+}$. Since $w_{\varepsilon}$ is continuous, for all $\varepsilon$ sufficiently small there exists $\delta_{\varepsilon} \in\left(\frac{r_{1}-r}{\varepsilon}, \frac{r_{2}-r}{\varepsilon}\right)$ such that

$$
w_{\varepsilon}\left(\delta_{\varepsilon}\right)=0 .
$$

Then $r_{1}-r \leq \varepsilon \delta_{\varepsilon} \leq r_{2}-r$ all $\varepsilon$ sufficiently small. Hence,

$$
r_{1}-r \leq \liminf _{\varepsilon \rightarrow 0^{+}} \varepsilon \delta_{\varepsilon} \leq \limsup _{\varepsilon \rightarrow 0^{+}} \varepsilon \delta_{\varepsilon} \leq r_{2}-r .
$$

Letting $r_{1} \rightarrow r^{-}$and $r_{2} \rightarrow r^{+}$, we conclude that (3.41) holds.

Step 8. Define

$$
\check{w}_{\varepsilon}(t):=w_{\varepsilon}\left(t+\delta_{\varepsilon}\right) \quad \text { for }-\frac{r}{\varepsilon}-\delta_{\varepsilon} \leq t \leq \frac{R-r}{\varepsilon}-\delta_{\varepsilon} .
$$

In this step we prove that

$$
\check{w}_{\varepsilon} \rightarrow z \text { strongly in } H^{1}(-b, b)
$$

for every $b>0$, where $z$ is the "optimal profile" introduced in (2.5).

Fix $0<r_{1}<r, r_{2}<R-r$, and $b \geq \tau_{W}$, where $\tau_{W}$ is given in (2.6). By (3.41), for all $\varepsilon>0$ sufficiently small so that $\left|\delta_{\varepsilon}\right|<\min \left\{\frac{r-r_{1}}{\varepsilon}, \frac{R-r-r_{2}}{\varepsilon}\right\}$, by (3.17), (3.41), and (3.42) we obtain

$$
\begin{aligned}
c_{W} r^{n-1} & +O\left(\varepsilon^{2}\right) \geq \mathcal{H}_{\varepsilon}\left(w_{\varepsilon}\right) \\
& =\int_{-\frac{r}{\varepsilon}-\delta_{\varepsilon}}^{\frac{R-r}{\varepsilon}-\delta_{\varepsilon}}\left(W\left(\check{w}_{\varepsilon}(s)\right)+\left|\check{w}_{\varepsilon}^{\prime}(s)\right|^{2}\right)\left(r+\varepsilon s+\varepsilon \delta_{\varepsilon}\right)^{n-1} d s \\
& \geq \int_{-\frac{r_{1}}{\varepsilon}}^{\frac{r_{2}}{\varepsilon}}\left(W\left(\check{w}_{\varepsilon}(s)\right)+\left|\check{w}_{\varepsilon}^{\prime}(s)\right|^{2}\right)\left(r+\varepsilon s+\varepsilon \delta_{\varepsilon}\right)^{n-1} d s \\
& \geq \int_{-b}^{b}\left(W\left(\check{w}_{\varepsilon}(s)\right)+\left|\check{w}_{\varepsilon}^{\prime}(s)\right|^{2}\right)\left(r+\varepsilon s+\varepsilon \delta_{\varepsilon}\right)^{n-1} d s .
\end{aligned}
$$

Fix $0<\eta<r$. Again by (3.41), $\left|\varepsilon s-\varepsilon \delta_{\varepsilon}\right| \leq \eta$ for all $\varepsilon$ sufficiently small, and so by (3.45),

$$
c_{W} r^{n-1}+\eta \geq(r-\eta)^{n-1} \int_{-b}^{b}\left(W\left(\check{w}_{\varepsilon}(s)\right)+\left|\check{w}_{\varepsilon}^{\prime}(s)\right|^{2}\right) d s .
$$


Since $\check{w}_{\varepsilon}(0)=0$, it follows that for all $\varepsilon$ sufficiently small the sequence $\left\{\check{w}_{\varepsilon}\right\}$ is bounded in $H^{1}(-b, b)$, and thus, up to a subsequence not relabeled, it converges weakly in $H^{1}(-b, b)$ and uniformly to some function $\check{w} \in H^{1}(-b, b)$. It follows by Fatou's Lemma and the weak sequential lower semicontinuity of the $L^{2}$ norm that

$$
\begin{aligned}
c_{W} r^{n-1}+\eta & \geq(r-\eta)^{n-1} \limsup _{\varepsilon \rightarrow 0^{+}} \int_{-b}^{b}\left(W\left(\check{w}_{\varepsilon}(s)\right)+\left|\check{w}_{\varepsilon}^{\prime}(s)\right|^{2}\right) d s \\
& \geq(r-\eta)^{n-1} \liminf _{\varepsilon \rightarrow 0^{+}} \int_{-b}^{b}\left(W\left(\check{w}_{\varepsilon}(s)\right)+\left|\check{w}_{\varepsilon}^{\prime}(s)\right|^{2}\right) d s \\
& \geq(r-\eta)^{n-1} \int_{-b}^{b}\left(W(\check{w}(s))+\left|\check{w}^{\prime}(s)\right|^{2}\right) d s
\end{aligned}
$$

Letting $\eta \rightarrow 0^{+}$gives

$$
\begin{aligned}
c_{W} & \geq \int_{-b}^{b}\left(W(\check{w}(s))+\left|\check{w}^{\prime}(s)\right|^{2}\right) d s \\
& \geq \min _{w \in H^{1}(-b, b), w(0)=0} \int_{-b}^{b}\left(W(w(s))+\left|w^{\prime}(s)\right|^{2}\right) d s=c_{W},
\end{aligned}
$$

where we have used (2.9). Since $\check{w}(0)=0$, from the uniqueness of the minimizer it follows that $\check{w}=z$. Hence, the entire sequence $\left\{\check{w}_{\varepsilon}\right\}$ weakly converges to $z$ in $H^{1}(-b, b)$, and by (3.46),

$$
c_{W}=\lim _{\varepsilon \rightarrow 0^{+}} \int_{-b}^{b}\left(W\left(\check{w}_{\varepsilon}(s)\right)+\left|\check{w}_{\varepsilon}^{\prime}(s)\right|^{2}\right) d s=\int_{-b}^{b}\left(W(z(s))+\left|z^{\prime}(s)\right|^{2}\right) d s
$$

which implies that

$$
\lim _{\varepsilon \rightarrow 0^{+}} \int_{-b}^{b}\left|\check{w}_{\varepsilon}^{\prime}(s)\right|^{2} d s=\int_{-b}^{b}\left|z^{\prime}(s)\right|^{2} d s
$$

and, in turn, (3.44) holds.

Step 9. Here we prove that the sequence of Lagrange multipliers $\lambda_{\varepsilon}$ found in Step 4 converges to $\lambda_{0}:=(n-1) c_{W}$, where $c_{W}$ is defined in (2.7).

Note that a similar result was obtained in [19] in the case of a mass equality constraint.

Since $\check{w}_{\varepsilon}\left(-\tau_{W}\right) \rightarrow-1$ and $\check{w}_{\varepsilon}\left(\tau_{W}\right) \rightarrow 1^{-}$by (3.44), there exists a sequence $\left\{\zeta_{\varepsilon}\right\}$ of positive numbers converging to 0 such that $1+\check{w}_{\varepsilon}\left(-\tau_{W}\right) \leq \zeta_{\varepsilon}$ and $1-\check{w}_{\varepsilon}\left(\tau_{W}\right) \leq \zeta_{\varepsilon}$ for every $\varepsilon>0$. Then for $\varepsilon>0$ small enough 


$$
\begin{aligned}
\int_{-\tau_{W}}^{\tau_{W}} & \left(W\left(\check{w}_{\varepsilon}(s)\right)+\left|\check{w}_{\varepsilon}^{\prime}(s)\right|^{2}\right)\left(r+\varepsilon s+\varepsilon \delta_{\varepsilon}\right)^{n-1} d s \\
& \geq 2\left(r-\varepsilon \tau_{W}+\varepsilon \delta_{\varepsilon}\right)^{n-1} \int_{-\tau_{W}}^{\tau_{W}}\left(\sqrt{W\left(\check{w}_{\varepsilon}(s)\right)} \check{w}_{\varepsilon}^{\prime}(s)\right) d s \\
& =2\left(r-\varepsilon \tau_{W}+\varepsilon \delta_{\varepsilon}\right)^{n-1} \int_{\check{w}_{\varepsilon}\left(-\tau_{W}\right)}^{\check{w}_{\varepsilon}\left(\tau_{W}\right)} \sqrt{W(\sigma)} d \sigma \\
& \geq 2\left(r-\varepsilon \tau_{W}+\varepsilon \delta_{\varepsilon}\right)^{n-1} \int_{-1}^{1} \sqrt{W(\sigma)} d \sigma \\
& -3 r^{n-1} \int_{1-\zeta_{\varepsilon}}^{1} \sqrt{W(\sigma)} d \sigma-3 r^{n-1} \int_{-1}^{-1+\zeta_{\varepsilon}} \sqrt{W(\sigma)} d \sigma .
\end{aligned}
$$

By (2.4) we have

$$
\int_{1-\zeta_{\varepsilon}}^{1} \sqrt{W(\sigma)} d \sigma=\int_{1-\zeta_{\varepsilon}}^{1}(1-\sigma)^{\beta / 2} d \sigma=\int_{0}^{\zeta_{\varepsilon}} s^{\beta / 2} d s=\frac{2}{2+\beta} \zeta_{\varepsilon}^{(2+\beta) / 2}
$$

and, similarly,

$$
\int_{-1}^{-1+\zeta_{\varepsilon}} \sqrt{W(\sigma)} d \sigma=\frac{2}{2+\beta} \zeta_{\varepsilon}^{(2+\beta) / 2}
$$

In conclusion,

$$
\begin{aligned}
\int_{-\tau_{W}}^{\tau_{W}} & \left(W\left(\check{w}_{\varepsilon}(s)\right)+\left|\check{w}_{\varepsilon}^{\prime}(s)\right|^{2}\right)\left(r+\varepsilon s+\varepsilon \delta_{\varepsilon}\right)^{n-1} d s \\
& \geq c_{W} r^{n-1}-O\left(\varepsilon \tau_{W}-\varepsilon \delta_{\varepsilon}\right)-\frac{12 r^{n-1}}{2+\beta} \zeta_{\varepsilon}^{(2+\beta) / 2} .
\end{aligned}
$$

Fix $0<r_{1}<r<r_{2}<R$. Using (3.45) and the previous inequality, we find that

$$
\begin{aligned}
\int_{\tau_{W}}^{\frac{r_{2}}{\varepsilon}} & \left(W\left(\check{w}_{\varepsilon}(s)\right)+\left|\check{w}_{\varepsilon}^{\prime}(s)\right|^{2}\right)\left(r+\varepsilon s+\varepsilon \delta_{\varepsilon}\right)^{n-1} d s \\
& +\int_{-\frac{r_{1}}{\varepsilon}}^{-\tau_{W}}\left(W\left(\check{w}_{\varepsilon}(s)\right)+\left|\check{w}_{\varepsilon}^{\prime}(s)\right|^{2}\right)\left(r+\varepsilon s+\varepsilon \delta_{\varepsilon}\right)^{n-1} d s \\
& \leq O\left(\varepsilon^{2}\right)+O\left(\varepsilon \tau_{W}-\varepsilon \delta_{\varepsilon}\right)+\frac{12 r^{n-1}}{2+\beta} \zeta_{\varepsilon}^{(2+\beta) / 2}=: \eta_{\varepsilon} .
\end{aligned}
$$

Note that, by (3.41),

$$
\lim _{\varepsilon \rightarrow 0+} \eta_{\varepsilon}=0
$$

Fix $0<r_{1}^{*}<r_{1}$ and $0<r<r_{2}^{*}<r_{2}$. We claim that there exist $a_{\varepsilon} \in$ $\left(-r_{1} / \varepsilon,-r_{1}^{*} / \varepsilon\right)$ and $b_{\varepsilon} \in\left(r_{2}^{*} / \varepsilon, r_{2} / \varepsilon\right)$ such that

$$
W\left(\check{w}_{\varepsilon}\left(a_{\varepsilon}\right)\right)+\left|\check{w}_{\varepsilon}^{\prime}\left(a_{\varepsilon}\right)\right|^{2} \leq c_{1} \varepsilon \eta_{\varepsilon} \quad \text { and } \quad W\left(\check{w}_{\varepsilon}\left(b_{\varepsilon}\right)\right)+\left|\check{w}_{\varepsilon}^{\prime}\left(b_{\varepsilon}\right)\right|^{2} \leq c \varepsilon \eta_{\varepsilon}
$$


for some appropriate constants $c_{1}=c_{1}\left(r_{1}^{*}, r_{1}\right)>0$ and $c_{2}=c_{2}\left(r_{2}^{*}, r_{2}\right)>0$, independent of $\varepsilon$. To prove the existence of $a_{\varepsilon}$, assume by contradiction that

$$
W\left(\check{w}_{\varepsilon}(s)\right)+\left|\check{w}_{\varepsilon}^{\prime}(s)\right|^{2}>c_{1} \varepsilon \eta_{\varepsilon}
$$

for all $s \in\left(-\frac{r_{1}}{\varepsilon},-\frac{r_{1}^{*}}{\varepsilon}\right)$. By (3.41), $\left|\varepsilon \delta_{\varepsilon}\right| \leq \frac{1}{2}\left(r-r_{1}\right)$ for all $\varepsilon$ sufficiently small, and so, by (3.47),

$$
\begin{aligned}
\eta_{\varepsilon} & \geq \int_{-\frac{r_{1}}{\varepsilon}}^{-\tau_{W}}\left(W\left(\check{w}_{\varepsilon}(s)\right)+\left|\check{w}_{\varepsilon}^{\prime}(s)\right|^{2}\right)\left(r+\varepsilon s+\varepsilon \delta_{\varepsilon}\right)^{n-1} d s \\
& \geq \int_{-\frac{r_{1}}{\varepsilon}}^{-\frac{r_{1}^{*}}{\varepsilon}}\left(W\left(\check{w}_{\varepsilon}(s)\right)+\left|\check{w}_{\varepsilon}^{\prime}(s)\right|^{2}\right)\left(r+\varepsilon s+\varepsilon \delta_{\varepsilon}\right)^{n-1} d s \\
& \geq c_{1} \eta_{\varepsilon}\left(r-r_{1}+\varepsilon \delta_{\varepsilon}\right)^{n-1}\left(r_{1}-r_{1}^{*}\right) \\
& \geq c_{1} \eta_{\varepsilon}\left(\frac{r-r_{1}}{2}\right)^{n-1}\left(r_{1}-r_{1}^{*}\right),
\end{aligned}
$$

which is a contradiction, provided we take

$$
c_{1}>\frac{2^{n-1}}{\left(r-r_{1}\right)^{n-1}\left(r_{1}-r_{1}^{*}\right)} .
$$

This proves the existence of $a_{\varepsilon}$. The proof of the existence of $b_{\varepsilon}$ is similar, and we omit it.

By (3.36), $\check{w}_{\varepsilon}$ satisfies the Euler-Lagrange equation

$$
-2 \check{w}_{\varepsilon}^{\prime \prime}(s)-2 \varepsilon(n-1) \frac{\check{w}_{\varepsilon}^{\prime}(s)}{r+\varepsilon s+\varepsilon \delta_{\varepsilon}}+W^{\prime}\left(\check{w}_{\varepsilon}(s)\right)=-\varepsilon \lambda_{\varepsilon} .
$$

Multiplying the previous equation by $\check{w}_{\varepsilon}^{\prime}(s)$ gives

$$
\left(-\left|\check{w}_{\varepsilon}^{\prime}(s)\right|^{2}+W\left(\check{w}_{\varepsilon}(s)\right)\right)^{\prime}=2 \varepsilon(n-1) \frac{\left|\check{w}_{\varepsilon}^{\prime}(s)\right|^{2}}{r+\varepsilon s+\varepsilon \delta_{\varepsilon}}-\varepsilon \lambda_{\varepsilon} \check{w}_{\varepsilon}^{\prime}(s) .
$$

Upon integration between $a_{\varepsilon}$ and $b_{\varepsilon}$ we get

$$
\begin{aligned}
& -\left|\check{w}_{\varepsilon}^{\prime}\left(b_{\varepsilon}\right)\right|^{2}+W\left(\check{w}_{\varepsilon}\left(b_{\varepsilon}\right)\right)+\left|\check{w}_{\varepsilon}^{\prime}\left(a_{\varepsilon}\right)\right|^{2}-W\left(\check{w}_{\varepsilon}\left(a_{\varepsilon}\right)\right) \\
& \quad=2 \varepsilon(n-1) \int_{a_{\varepsilon}}^{b_{\varepsilon}} \frac{\left|\check{w}_{\varepsilon}^{\prime}(s)\right|^{2}}{r+\varepsilon s+\varepsilon \delta_{\varepsilon}} d s-\varepsilon \lambda_{\varepsilon}\left(\check{w}_{\varepsilon}\left(b_{\varepsilon}\right)-\check{w}_{\varepsilon}\left(a_{\varepsilon}\right)\right) .
\end{aligned}
$$

Since $a_{\varepsilon} \in\left(-r_{1} / \varepsilon,-r_{1}^{*} / \varepsilon\right)$ and $b_{\varepsilon} \in\left(r_{2}^{*} / \varepsilon, r_{2} / \varepsilon\right)$ it follows from (3.21) and the monotonicity of $\check{w}_{\varepsilon}$ that $\check{w}_{\varepsilon}\left(a_{\varepsilon}\right) \rightarrow-1$ and $\check{w}_{\varepsilon}\left(b_{\varepsilon}\right) \rightarrow 1$. Dividing the previous identity by $\varepsilon$ and letting $\varepsilon \rightarrow 0^{+}$, by (3.49) we get

$$
\lim _{\varepsilon \rightarrow 0^{+}}\left(2(n-1) \int_{a_{\varepsilon}}^{b_{\varepsilon}} \frac{\left|\check{w}_{\varepsilon}^{\prime}(s)\right|^{2}}{r+\varepsilon s+\varepsilon \delta_{\varepsilon}} d s-\lambda_{\varepsilon}\right)=0 .
$$


Observe that by (3.47),

$$
\begin{aligned}
\int_{\tau_{W}}^{b_{\varepsilon}} \frac{\left|\check{w}_{\varepsilon}^{\prime}(s)\right|^{2}}{r+\varepsilon s+\varepsilon \delta_{\varepsilon}} d s & \leq \frac{1}{\left(r+\varepsilon \delta_{\varepsilon}\right)^{n}} \int_{\tau_{W}}^{b_{\varepsilon}}\left|\check{w}_{\varepsilon}^{\prime}(s)\right|^{2}\left(r+\varepsilon s+\varepsilon \delta_{\varepsilon}\right)^{n-1} d s \\
& \leq \frac{\eta_{\varepsilon}}{\left(r+\varepsilon \delta_{\varepsilon}\right)^{n}} \rightarrow 0
\end{aligned}
$$

where the convergence to zero follows from (3.41) and (3.48). Similarly,

$$
\int_{a_{\varepsilon}}^{-\tau_{W}} \frac{\left|\check{w}_{\varepsilon}^{\prime}(s)\right|^{2}}{r+\varepsilon s+\varepsilon \delta_{\varepsilon}} d s \leq \frac{\eta_{\varepsilon}}{\left(r-r_{1}+\varepsilon \delta_{\varepsilon}\right)^{n}} \rightarrow 0 .
$$

These inequalities, together with (3.44) and (3.51), imply that

$$
\lim _{\varepsilon \rightarrow 0^{+}} \lambda_{\varepsilon}=\lambda_{0}:=2(n-1) \int_{-\tau_{W}}^{\tau_{W}}\left|z^{\prime}(s)\right|^{2} d s=(n-1) c_{W},
$$

where in the last equality we have used (2.8).

Step 10. We claim that there exist two constants $a_{1}>0$ and $c_{1}>0$ such that

$$
\check{w}_{\varepsilon}\left(-\tau_{W}-a_{1} \varepsilon\right) \leq-1+c_{1} \varepsilon^{1 / \beta}
$$

for all $\varepsilon>0$ sufficiently small.

Let $b_{\varepsilon}$ be the number given in (3.49) and let $t<b_{\varepsilon}$. Integrating (3.50) between $t$ and $b_{\varepsilon}$ and using (3.35) and (3.52) gives

$$
\begin{aligned}
& -\left|\check{w}_{\varepsilon}^{\prime}\left(b_{\varepsilon}\right)\right|^{2}+W\left(\check{w}_{\varepsilon}\left(b_{\varepsilon}\right)\right)+\left|\check{w}_{\varepsilon}^{\prime}(t)\right|^{2}-W\left(\check{w}_{\varepsilon}(t)\right) \\
& =2 \varepsilon(n-1) \int_{t}^{b_{\varepsilon}} \frac{\left|\check{w}_{\varepsilon}^{\prime}(s)\right|^{2}}{r+\varepsilon s-\varepsilon \delta_{\varepsilon}} d s-\varepsilon \lambda_{\varepsilon}\left(\check{w}_{\varepsilon}\left(b_{\varepsilon}\right)-\check{w}_{\varepsilon}(t)\right) \geq-c \varepsilon
\end{aligned}
$$

for some constant $c>0$ independent of $\varepsilon$. In view of (3.49), by taking $c>0$ larger, if necessary, we have

$$
\left|\check{w}_{\varepsilon}^{\prime}(t)\right|^{2}>W\left(\check{w}_{\varepsilon}(t)\right)-c \varepsilon .
$$

Since $\check{w}_{\varepsilon}$ is nondecreasing, we obtain that

$$
\check{w}_{\varepsilon}^{\prime}(t) \geq \sqrt{\left(W\left(\check{w}_{\varepsilon}(t)\right)-c \varepsilon\right)^{+}} .
$$

Using the fact that $W$ is continuous and vanishes only at $s= \pm 1$, we find a constant $\mu>0$ such that

$$
W(s) \geq \mu \text { for }-1+a \leq s \leq 1-a,
$$

where $a$ is the constant in (2.4). Therefore, if $c \varepsilon<\mu$ and $(c \varepsilon)^{1 / \beta}<a$ we deduce from (2.4) that $W(s)>c \varepsilon$ for $-1+(c \varepsilon)^{1 / \beta}<s \leq 0$. This implies

$$
\check{w}_{\varepsilon}^{\prime}(t) \geq \sqrt{W\left(\check{w}_{\varepsilon}(t)\right)-c \varepsilon} .
$$


for every $-\frac{r}{\varepsilon}-\delta_{\varepsilon}<t \leq 0$ such that $-1+(c \varepsilon)^{1 / \beta}<\check{w}_{\varepsilon}(t) \leq 0$.

Since $\check{w}_{\mathcal{\varepsilon}}(0)=0$, it follows upon integration

$$
-t \leq \int_{\check{w}_{\varepsilon}(t)}^{0} \frac{1}{\sqrt{W(s)-c \varepsilon}} d s \leq \int_{-1+(c \varepsilon)^{1 / \beta}}^{0} \frac{1}{\sqrt{W(s)-c \varepsilon}} d s<+\infty,
$$

where the last inequality is a consequence of (2.4). Observe that this inequality provides a bound on all those $t$ such that $-1+(c \varepsilon)^{1 / \beta}<\check{w}_{\varepsilon}(t) \leq 0$. Therefore, there exists a largest $t_{\varepsilon}$ such that $-\frac{r}{\varepsilon}-\delta_{\varepsilon}<t_{\varepsilon}<0$ and $\check{w}_{\varepsilon}\left(t_{\varepsilon}\right)=-1+(c \varepsilon)^{1 / \beta}$. Then

$$
\begin{aligned}
-t_{\varepsilon} & \leq \int_{-1+(c \varepsilon)^{1 / \beta}}^{0} \frac{1}{\sqrt{W(s)-c \varepsilon}} d s \\
& =\int_{-1+(c \varepsilon)^{1 / \beta}}^{-1+a} \frac{1}{\sqrt{(s+1)^{\beta}-c \varepsilon}} d s+\int_{-1+a}^{0} \frac{1}{\sqrt{W(s)-c \varepsilon}} d s .
\end{aligned}
$$

The change of variables $\sigma:=(s+1)^{\beta}-c \varepsilon$ yields

$$
\begin{aligned}
& \int_{-1+(c \varepsilon)^{1 / \beta}}^{-1+a} \frac{1}{\sqrt{(s+1)^{\beta}-c \varepsilon}} d s=\frac{1}{\beta} \int_{0}^{a^{\beta}-c \varepsilon} \frac{1}{\sigma^{1 / 2}(\sigma+c \varepsilon)^{1-1 / \beta}} d \sigma \\
& <\frac{1}{\beta} \int_{0}^{a^{\beta}} \frac{1}{\sigma^{1 / 2} \sigma^{1-1 / \beta}} d \sigma=\int_{-1}^{-1+a} \frac{1}{\sqrt{W(s)}} d s
\end{aligned}
$$

where the last equality follows from direct computation, taking into account (2.4).

By (3.54) there exists a constant $L>0$ such that

$$
\left|\frac{1}{\sqrt{W(s)-\eta}}-\frac{1}{\sqrt{W(s)}}\right| \leq L \eta
$$

for $-1+a \leq s \leq 1-a$ and for $0<\eta \leq \mu / 2$.

From (3.55), (3.56), and (3.57) we get

$$
-t_{\varepsilon} \leq \int_{-1}^{0} \frac{d s}{\sqrt{W(s)}}+L c \varepsilon=\tau_{W}+L c \varepsilon
$$

where the equality follows from (2.6). Since $\check{w}_{\varepsilon}$ is nondecreasing, we obtain $\check{w}_{\varepsilon}\left(-\tau_{W}-L c \varepsilon\right) \leq \check{w}_{\varepsilon}\left(t_{\varepsilon}\right)=-1+(c \varepsilon)^{1 / \beta}$, which gives (3.53) with $a_{1}:=L c$ and $c_{1}:=c^{1 / \beta}$.

Step 11. Let $1<\alpha \leq 2$ be defined by

$$
\alpha:=\frac{1}{\frac{1}{2}+\left|\beta-\frac{3}{2}\right|} .
$$


We claim there exist two constants $a_{2}>0$ and $c_{2}>0$ such that

$$
\check{w}_{\varepsilon}\left(\tau_{W}+a_{2} \varepsilon\right) \geq 1-c_{2} \varepsilon^{\alpha}
$$

for all $\varepsilon>0$ sufficiently small.

Since $\check{w}_{\varepsilon}\left(\frac{R-r}{\varepsilon}-\delta_{\varepsilon}\right)=1$, integrating (3.50) between $t$ and $\frac{R-r}{\varepsilon}-\delta_{\varepsilon}$ we obtain

$$
\begin{aligned}
& \left|\check{w}_{\varepsilon}^{\prime}(t)\right|^{2}-W\left(\check{w}_{\varepsilon}(t)\right)+\varepsilon \lambda_{\varepsilon}\left(1-\check{w}_{\varepsilon}(t)\right) \\
& =\left|\check{w}_{\varepsilon}^{\prime}\left(\frac{R-r}{\varepsilon}-\delta_{\varepsilon}\right)\right|^{2}+2 \varepsilon(n-1) \int_{t}^{\frac{R-r}{\varepsilon}-\delta_{\varepsilon}} \frac{\left|\check{w}_{\varepsilon}^{\prime}(s)\right|^{2}}{r+\varepsilon s+\varepsilon \delta_{\varepsilon}} d s \geq 0 .
\end{aligned}
$$

Hence, by (3.52)

$$
\left|\check{w}_{\varepsilon}^{\prime}(t)\right|^{2} \geq W\left(\check{w}_{\varepsilon}(t)\right)-c \varepsilon\left(1-\check{w}_{\varepsilon}(t)\right)
$$

for all $\varepsilon>0$ sufficiently small and for some constant $c>0$ independent of $\varepsilon$. Since $\check{w}_{\varepsilon}$ is nondecreasing, we deduce that

$$
\check{w}_{\varepsilon}^{\prime}(t) \geq \sqrt{\left.\left(W \check{w}_{\varepsilon}(t)\right)-c \varepsilon\left(1-\check{w}_{\varepsilon}(t)\right)\right)^{+}} .
$$

For $t>0$ sufficiently small, we have that $0 \leq \check{w}_{\varepsilon}(t)<1$. Since $\check{w}_{\varepsilon}(0)=0$, it follows upon integration

$$
t \leq \int_{0}^{\check{w}_{\varepsilon}(t)} \frac{d s}{\sqrt{(W(s)-c \varepsilon(1-s))^{+}}} .
$$

Let $\gamma:=\min \{2(\beta-1), 1\}$. Note that $\alpha=\frac{1}{\beta-\gamma}$. Then $(W(s)-c \varepsilon(1-s))^{+} \geq$ $\left(W(s)-c \varepsilon(1-s)^{\gamma}\right)^{+}$for $0 \leq s \leq 1$, hence

$$
t \leq \int_{0}^{\check{w}_{\varepsilon}(t)} \frac{d s}{\sqrt{\left(W(s)-c \varepsilon(1-s)^{\gamma}\right)^{+}}} .
$$

By (3.54) when $c \varepsilon \leq \mu$ we have $W(s)-c \varepsilon(1-s)^{\gamma} \geq 0$ for $0 \leq s \leq 1-a$. Moreover, if $(c \varepsilon)^{\alpha}<a$ we obtain $W(s)=(1-s)^{\beta}$ for $1-a \leq s \leq 1-(c \varepsilon)^{\alpha}$ by (2.4).

Using the facts that $\check{w}_{\varepsilon}(0)=0$ and $\check{w}_{\varepsilon}\left(\frac{R-r}{\varepsilon}-\delta_{\varepsilon}\right)=1$, there exists $0<t_{\varepsilon}<$ $\frac{R-r}{\varepsilon}-\delta_{\varepsilon}$ such that $\check{w}_{\varepsilon}\left(t_{\varepsilon}\right)=1-(c \varepsilon)^{\alpha}$. Then for $\varepsilon$ sufficiently small

$$
t_{\varepsilon} \leq \int_{0}^{1-a} \frac{d s}{\sqrt{W(s)-c \varepsilon(1-s)^{\gamma}}}+\int_{1-a}^{1-(c \varepsilon)^{\alpha}} \frac{d s}{(1-s)^{\gamma / 2} \sqrt{(1-s)^{\beta-\gamma}-c \varepsilon}} .
$$


Consider the change of variables $\sigma:=(1-s)^{\beta-\gamma}-c \varepsilon$. Then $(\sigma+c \varepsilon)^{1 /(\beta-\gamma)}=$ $1-s$, and since $2-2 \beta+\gamma \leq 0$ and $\beta-\gamma \geq 0$,

$$
\begin{aligned}
& \int_{1-a}^{1-(c \varepsilon)^{\alpha}} \frac{d s}{(1-s)^{\gamma / 2} \sqrt{(1-s)^{\beta-\gamma}-c \varepsilon}} \\
& \leq \frac{1}{\beta-\gamma} \int_{0}^{a^{\beta-\gamma}-c \varepsilon}(\sigma+c \varepsilon)^{(2-2 \beta+\gamma) /(2 \beta-2 \gamma)} \sigma^{-1 / 2} d \sigma \\
& \leq \frac{1}{\beta-\gamma} \int_{0}^{a^{\beta-\gamma}} \sigma^{(2-2 \beta+\gamma) /(2 \beta-2 \gamma)} \sigma^{-1 / 2} d \sigma \\
& =\frac{2}{2-\beta} a^{\frac{2-\beta}{2}}=\int_{1-a}^{1} \frac{d s}{\sqrt{W(s)}}
\end{aligned}
$$

where the last inequality follows from direct computation, taking into account (2.4).

From (3.57), (3.59), and (3.60) we obtain that for $\varepsilon>0$ small enough we have

$$
t_{\varepsilon} \leq \int_{0}^{1} \frac{d s}{\sqrt{W(s)}}+L c \varepsilon=\tau_{W}+L c \varepsilon,
$$

where the equality is a consequence of (2.6). Since $\check{w}_{\varepsilon}$ is nondecreasing, it follows that $\check{w}_{\varepsilon}\left(\tau_{W}+L c \varepsilon\right) \geq \check{w}_{\varepsilon}\left(t_{\varepsilon}\right)=1-(c \varepsilon)^{\alpha}$. This concludes the proof of (3.58) with $a_{2}:=L c$ and $c_{2}:=c^{\alpha}$.

Step 12. Let $\delta_{\varepsilon}$ be the constant introduced in Step 7 and let $w_{0}$ be the function introduced in (3.38). We claim that

$$
\liminf _{\varepsilon \rightarrow 0+} \int_{\delta_{\varepsilon}+\tau_{W}}^{\frac{R-r}{\varepsilon}}\left(w_{\varepsilon}(t)-w_{0}(t)\right)(r+\varepsilon t)^{n-1} d t \geq 0 .
$$

Since $w_{\varepsilon}$ is nondecreasing and $w_{\varepsilon}\left(\delta_{\varepsilon}+\tau_{W}+a_{2} \varepsilon\right) \geq 1-c_{2} \varepsilon^{\alpha}$ by (3.58), we have $w_{\varepsilon}(t) \geq 1-c_{2} \varepsilon^{\alpha}>0$ for every $t \geq \delta_{\varepsilon}+\tau_{W}+a_{2} \varepsilon$. This implies that

$$
\begin{aligned}
& \int_{\delta_{\varepsilon}+\tau_{W}}^{\frac{R-r}{\varepsilon}}\left(w_{\varepsilon}(t)-w_{0}(t)\right)(r+\varepsilon t)^{n-1} d t=\int_{\delta_{\varepsilon}+\tau_{W}}^{\delta_{\varepsilon}+\tau_{W}+a_{2} \varepsilon}\left(w_{\varepsilon}(t)-w_{0}(t)\right)(r+\varepsilon t)^{n-1} d t \\
& +\int_{\delta_{\varepsilon}+\tau_{W}+a_{2} \varepsilon}^{\left(\delta_{\varepsilon}+\tau_{W}+a_{2} \varepsilon\right)^{+}}\left(w_{\varepsilon}(t)+1\right)(r+\varepsilon t)^{n-1} d t+\int_{\left(\delta_{\varepsilon}+\tau_{W}+a_{2} \varepsilon\right)^{+}}^{\frac{R-r}{\varepsilon}}\left(w_{\varepsilon}(t)-1\right)(r+\varepsilon t)^{n-1} d t \\
& \geq-c_{2} \varepsilon^{\alpha} \int_{\left(\delta_{\varepsilon}+\tau_{W}\right)^{+}}^{\frac{R-r}{\varepsilon}}(r+\varepsilon t)^{n-1} d t+O(\varepsilon) \geq-c_{2} \varepsilon^{\alpha-1} \frac{R^{n}-r^{n}}{n}+O(\varepsilon)
\end{aligned}
$$

where we used (3.41) together with the inequality

$$
\left.\int_{\delta_{\varepsilon}+\tau_{W}}^{\delta_{\varepsilon}+\tau_{W}+a_{2} \varepsilon}(t)-w_{0}(t)\right)(r+\varepsilon t)^{n-1} d t \geq-3\left(r+\varepsilon\left(\left|\delta_{\varepsilon}\right|+\tau_{W}+a_{2} \varepsilon\right)\right)^{n-1} a_{2} \varepsilon,
$$


which follows from (3.35).

Since $\alpha>1$, inequality (3.62) yields (3.61).

Step 13. We claim that

$$
\liminf _{\varepsilon \rightarrow 0+} \delta_{\varepsilon} \geq 0 .
$$

Assume, by contradiction, that there exists a sequence $\varepsilon_{j} \rightarrow 0+$ such that $\delta_{\varepsilon_{j}} \rightarrow \delta_{0}$ for some $\delta_{0}$ satisfying $-\infty \leq \delta_{0}<0$. This implies, in particular, that $\delta_{\varepsilon_{j}}-\tau_{W}<0$ for $j$ large enough. By (3.29) it follows that

$$
\begin{aligned}
& \int_{-\frac{r}{\varepsilon_{j}}}^{\delta_{\varepsilon_{j}}-\tau_{W}}\left(w_{\varepsilon_{j}}(t)-w_{0}(t)\right)\left(r+\varepsilon_{j} t\right)^{n-1} d t=\int_{-\frac{r}{\varepsilon_{j}}}^{\delta_{\varepsilon_{j}}-\tau_{W}}\left(w_{\varepsilon_{j}}(t)+1\right)\left(r+\varepsilon_{j} t\right)^{n-1} d t \\
& \geq-\left(\frac{\varepsilon_{j} \lambda_{\varepsilon_{j}}}{\beta}\right)^{1 /(\beta-1)} \frac{r^{n}}{n \varepsilon_{j}}=-\frac{\varepsilon_{j}^{(2-\beta) /(\beta-1)} \lambda_{\varepsilon_{j}}^{1 /(\beta-1)} r^{n}}{\beta^{1 /(\beta-1)} n} .
\end{aligned}
$$

Since $1<\beta<2$, by (3.52) we obtain

$$
\liminf _{j \rightarrow \infty} \int_{-\frac{r}{\varepsilon_{j}}}^{\delta_{\varepsilon_{j}}-\tau_{W}}\left(w_{\varepsilon_{j}}(t)-w_{0}(t)\right)\left(r+\varepsilon_{j} t\right)^{n-1} d t \geq 0 .
$$

This inequality, together with (3.39) and (3.61), implies that

$$
\limsup _{j \rightarrow \infty} \int_{\delta_{\varepsilon_{j}}-\tau_{W}}^{\delta_{\varepsilon_{j}}+\tau_{W}}\left(w_{\varepsilon_{j}}(t)-w_{0}(t)\right)\left(r+\varepsilon_{j} t\right)^{n-1} d t \leq 0 .
$$

Changing variables, we get

$$
\limsup _{j \rightarrow \infty} \int_{-\tau_{W}}^{\tau_{W}}\left(\check{w}_{\varepsilon_{j}}(t)-w_{0}\left(t+\delta_{\varepsilon_{j}}\right)\right)\left(r+\varepsilon_{j} t+\varepsilon_{j} \delta_{\varepsilon_{j}}\right)^{n-1} d t \leq 0 .
$$

Since $\varepsilon_{j} \delta_{\varepsilon_{j}} \rightarrow 0$ by (3.41), we conclude that

$$
\limsup _{j \rightarrow \infty} \int_{-\tau_{W}}^{\tau_{W}}\left(\check{w}_{\varepsilon_{j}}(t)-w_{0}\left(t+\delta_{\varepsilon_{j}}\right)\right) d t \leq 0 .
$$

Using the fact that $\check{w}_{\varepsilon_{j}} \rightarrow z$ strongly in $H^{1}\left(-\tau_{W}, \tau_{W}\right)$, from the previous inequality we obtain

$$
\int_{-\tau_{W}}^{\tau_{W}}\left(z(t)-w_{0}\left(t+\hat{\delta}_{0}\right)\right) d t \leq 0
$$

where $\hat{\delta}_{0}:=\max \left\{\delta_{0},-\tau_{W}\right\}$. Indeed, if $\delta_{0} \geq-\tau_{W}$ we pass to the limit in (3.64). If $-\infty<\delta_{0}<-\tau_{W}$ we use also the equalities $w_{0}\left(t+\delta_{0}\right)=w_{0}\left(t+\hat{\delta}_{0}\right)=-1$ for $t \in\left[-\tau_{W}, \tau_{W}\right]$ in the case $\delta_{0}<-\tau_{W}$. The case $\delta_{0}=-\infty$ is similar. Since

$$
\int_{-\tau_{W}}^{\tau_{W}} z(t) d t=0 \quad \text { and } \quad \int_{-\tau_{W}}^{\tau_{W}} w_{0}\left(t+\hat{\delta}_{0}\right) d t=2 \hat{\delta}_{0},
$$


from (3.65) we obtain $2 \hat{\delta}_{0} \geq 0$, which contradicts the inequality $\delta_{0}<0$. This completes the proof of (3.63).

Step 14. We conclude the proof of the theorem by showing that (3.2) holds.

Let $a_{1}$ and $a_{2}$ be the constants given in (3.57) and (3.58). By (3.3), (3.41), and (3.43) we have

$$
\begin{aligned}
\mathcal{H}_{\varepsilon}\left(w_{\varepsilon}\right) & \geq \int_{\delta_{\varepsilon}-\tau_{W}-a_{1} \varepsilon}^{\delta_{\varepsilon}+\tau_{W}+a_{2} \varepsilon}\left(W\left(w_{\varepsilon}(t)\right)+\left|w_{\varepsilon}^{\prime}(t)\right|^{2}\right)(r+\varepsilon t)^{n-1} d t \\
& =\int_{-\tau_{W}-a_{1} \varepsilon}^{\tau_{W}+a_{2} \varepsilon}\left(W\left(\check{w}_{\varepsilon}(s)\right)+\left|\check{w}_{\varepsilon}^{\prime}(s)\right|^{2}\right)\left(r+\varepsilon s+\varepsilon \delta_{\varepsilon}\right)^{n-1} d s \\
& \geq I_{\varepsilon}^{1}+\varepsilon I_{\varepsilon}^{2}+\varepsilon \delta_{\varepsilon} I_{\varepsilon}^{3}+\varepsilon I_{\varepsilon}^{4},
\end{aligned}
$$

where

$$
\begin{aligned}
I_{\varepsilon}^{1} & :=2 r^{n-1} \int_{-\tau_{W}-a_{1} \varepsilon}^{\tau_{W}+a_{2} \varepsilon} \sqrt{W\left(\check{w}_{\varepsilon}(s)\right)}\left|\check{w}_{\varepsilon}^{\prime}(s)\right| d s, \\
I_{\varepsilon}^{2} & :=(n-1) r^{n-2} \int_{-\tau_{W}-a_{1} \varepsilon}^{\tau_{W}+a_{2} \varepsilon}\left(W\left(\check{w}_{\varepsilon}(s)\right)+\left|\check{w}_{\varepsilon}^{\prime}(s)\right|^{2}\right) s d s, \\
I_{\varepsilon}^{3} & :=(n-1) r^{n-2} \int_{-\tau_{W}-a_{1} \varepsilon}^{\tau_{W}+a_{2} \varepsilon}\left(W\left(\check{w}_{\varepsilon}(s)\right)+\left|\check{w}_{\varepsilon}^{\prime}(s)\right|^{2}\right) d s, \\
I_{\varepsilon}^{4} & :=\sum_{h=2}^{n-1}\left(\begin{array}{c}
n-1 \\
h
\end{array}\right) r^{n-1-h} \varepsilon^{h-1} J_{h, \varepsilon},
\end{aligned}
$$

with

$$
J_{h, \varepsilon}:=\int_{-\tau_{W}-a_{1} \varepsilon}^{\tau_{W}+a_{2} \varepsilon}\left(W\left(\check{w}_{\varepsilon}(s)\right)+\left|\check{w}_{\varepsilon}^{\prime}(s)\right|^{2}\right)\left(s+\delta_{\varepsilon}\right)^{h} d s .
$$

To estimate $I_{\varepsilon}^{1}$ we use (1.8) to write

$$
\begin{aligned}
I_{\varepsilon}^{1} & =2 r^{n-1} \int_{\check{w}_{\varepsilon}\left(-\tau_{W}-a_{1} \varepsilon\right)}^{\check{w}_{\varepsilon}\left(\tau_{W}+a_{2} \varepsilon\right)} \sqrt{W(\sigma)} d \sigma \\
& =c_{W} r^{n-1}-2 r^{n-1} \int_{-1}^{\check{w}_{\varepsilon}\left(-\tau_{W}-a_{1} \varepsilon\right)} \sqrt{W(\sigma)} d \sigma-2 r^{n-1} \int_{\check{w}_{\varepsilon}\left(\tau_{W}+a_{2} \varepsilon\right)}^{1} d \sigma .
\end{aligned}
$$

By (2.4) and (3.53) we have

$$
\begin{aligned}
& 2 r^{n-1} \int_{-1}^{\check{w}_{\varepsilon}\left(-\tau_{W}-a_{1} \varepsilon\right)} \sqrt{W(\sigma)} d \sigma \leq 2 r^{n-1} \int_{-1}^{-1+c_{1} \varepsilon^{1 / \beta}} \sqrt{W(\sigma)} d \sigma \\
& =2 r^{n-1} \int_{0}^{c_{1} \varepsilon^{1 / \beta}} s^{\beta / 2} d s=k_{1} \varepsilon^{(\beta+2) /(2 \beta)}
\end{aligned}
$$


where $k_{1}:=\frac{4}{\beta+2} r^{n-1} c_{1}^{(\beta+2) / 2}$. Similarly, by (3.58) we can find a constant $k_{2}>0$ such that

$$
\int_{\check{w}_{\varepsilon}\left(\tau_{W}+a_{2} \varepsilon\right)}^{1} \sqrt{W(\sigma)} d \sigma \leq k_{2} \varepsilon^{(\beta+2) /(2 \beta)},
$$

where we have used the fact that $\alpha>1 / \beta$.

Therefore (3.67) gives

$$
I_{\varepsilon}^{1} \geq c_{W} r^{n-1}-k_{0} \varepsilon^{(\beta+2) /(2 \beta)}
$$

where $k_{0}:=k_{1}+k_{2}$.

By (2.7), (3.35), and (3.44) we have

$$
\begin{aligned}
& \lim _{\varepsilon \rightarrow 0+} I_{\varepsilon}^{2}=(n-1) r^{n-2} \int_{-\tau_{W}}^{\tau_{W}}\left(W(z(s))+\left|z^{\prime}(s)\right|^{2}\right) s d s=0, \\
& \lim _{\varepsilon \rightarrow 0+} I_{\varepsilon}^{3}=(n-1) r^{n-2} \int_{-\tau_{W}}^{\tau_{W}}\left(W(z(s))+\left|z^{\prime}(s)\right|^{2}\right) d s=(n-1) c_{W} r^{n-2},
\end{aligned}
$$

where to obtain the second equality in (3.69) we have used the fact that $z$ is odd and $W$ is even .

Moreover, $J_{h, \varepsilon} \geq 0$ if $h$ is even, while if $h$ is odd by Fatou's Lemma, (3.44), and (3.63) we obtain

$$
\liminf _{\varepsilon \rightarrow 0+} J_{h, \varepsilon} \geq \int_{-\tau_{W}}^{\tau_{W}}\left(W(z(s))+\left|z^{\prime}(s)\right|^{2}\right) s^{h} d s=0,
$$

and the integral is zero because $z$ is odd and $W$ is even. Hence,

$$
\liminf _{\varepsilon \rightarrow 0+} I_{\varepsilon}^{4} \geq 0 .
$$

From (3.66) and (3.68) we obtain

$$
\frac{\mathcal{H}_{\varepsilon}\left(w_{\varepsilon}\right)-c_{W} r^{n-1}}{\varepsilon} \geq-k_{0} \varepsilon^{(2-\beta) /(2 \beta)}+I_{\varepsilon}^{2}+\delta_{\varepsilon} I_{\varepsilon}^{3}+I_{\varepsilon}^{4} .
$$

Since $1<\beta<2$, using (3.63), (3.69), (3.70), and (3.71) we conclude that (3.2) holds.

\section{The Limsup Inequality}

In this section we prove the following theorem.

Theorem 4.1 Let $E_{0}$ be a solution to the minimum problem (2.17) and let $u_{0}:=1-2 \chi_{E_{0}}$. Then there exists a sequence $\left\{u_{\varepsilon}\right\} \subset H^{1}(\Omega)$ converging to $u_{0}$ strongly in $L^{1}(\Omega)$ and satisfying (1.2) such that

$$
\limsup _{\varepsilon \rightarrow 0+} \frac{\mathcal{E}_{\varepsilon}\left(u_{\varepsilon}\right)-n \kappa_{\Phi} c_{W} r^{n-1}}{\varepsilon} \leq 0 .
$$


Note that by (2.25) the inequality (4.1) is equivalent to (1.11).

Proof. As observed in in Section 2, the set $E_{0}$ has the form $B_{r}^{\Phi^{\circ}}\left(x_{0}\right)$, with $B_{r}^{\Phi^{\circ}}\left(x_{0}\right) \subset \Omega$ and $r$ defined by (2.18). We recall that by hypothesis (2.21) there exist $y_{0} \in \Omega$ and $\delta>0$ satisfying $B_{r}^{\Phi^{\circ}}\left(x_{0}\right) \subset B_{r+\delta}^{\Phi^{\circ}}\left(y_{0}\right) \subset \Omega$.

We claim that $\Phi^{\circ}\left(x_{0}-y_{0}\right) \leq \delta$. If $\Phi^{\circ}\left(x_{0}-y_{0}\right)=0$ then the inequality is trivial. If not, let $\left\{\lambda_{k}\right\}$ be an increasing sequence converging to $r / \Phi^{\circ}\left(x_{0}-y_{0}\right)$. Since $x_{0}+\lambda_{k}\left(x_{0}-y_{0}\right) \in B_{r}^{\Phi^{\circ}}\left(x_{0}\right)$, we have $x_{0}+\lambda_{k}\left(x_{0}-y_{0}\right) \in B_{r+\delta}^{\Phi^{\circ}}\left(y_{0}\right)$, hence $\left(1+\lambda_{k}\right) \Phi^{\circ}\left(x_{0}-y_{0}\right) \leq r+\delta$. Passing to the limit as $k \rightarrow+\infty$, we obtain $\Phi^{\circ}\left(x_{0}-y_{0}\right)+r \leq r+\delta$, which implies $\Phi^{\circ}\left(x_{0}-y_{0}\right) \leq \delta$.

Since $\Phi^{\circ}$ is convex and positively homogeneous of degree one, it is subadditive and so the previous inequality gives

$$
B_{r+t \delta}^{\Phi^{\circ}}\left(x_{0}+t\left(y_{0}-x_{0}\right)\right) \subset B_{r+\delta}^{\Phi^{\circ}}\left(y_{0}\right) \subset \Omega
$$

for every $0 \leq t \leq 1$.

Let $z$ be the function defined by (2.5). We recall that $z$ is odd, $|z(t)| \leq 1$ for every $t \in \mathbb{R}$, and $z(t)=1$ if $t \geq \tau_{W}$, where $\tau_{W}$ is defined by (2.6). Let

$$
\hat{u}_{\varepsilon}(x):=z\left(\frac{\Phi^{\circ}\left(x-x_{0}-\varepsilon \gamma\left(y_{0}-x_{0}\right)\right)-r}{\varepsilon}\right) \quad \text { for } x \in \Omega,
$$

where $\gamma:=\tau_{W} / \delta$. Then $\hat{u}_{\varepsilon} \in H^{1}(\Omega)$ and $\hat{u}_{\varepsilon} \rightarrow u_{0}=1-2 \chi_{B_{r}^{\Phi^{\circ}}\left(x_{0}\right)}$ strongly in $L^{1}(\Omega)$. Since $\hat{u}_{\varepsilon}=1$ on $\Omega \backslash B_{r+\varepsilon \tau_{W}}^{\Phi^{\circ}}\left(x_{0}+\varepsilon \gamma\left(y_{0}-x_{0}\right)\right)$, it follows from (4.2) that $\hat{u}_{\varepsilon}=1$ on $\partial \Omega$ for $\varepsilon>0$ sufficiently small.

Observe that $\hat{u}_{\varepsilon}$ may not satisfy the mass constraint in (1.2), and so we estimate the possible error

$$
\omega_{\varepsilon}:=\int_{\Omega} \hat{u}_{\varepsilon}(x) d x-m .
$$

Using the fact that $\hat{u}_{\varepsilon}=-1$ on $B_{r-\varepsilon \tau_{W}}^{\Phi^{\circ}}\left(x_{0}+\varepsilon \gamma\left(y_{0}-x_{0}\right)\right)$, by (2.14) and (2.15) we get

$$
\begin{aligned}
\omega_{\varepsilon}= & \left|\Omega \backslash B_{r+\varepsilon \tau_{W}}^{\Phi^{\circ}}\left(x_{0}+\varepsilon \gamma\left(y_{0}-x_{0}\right)\right)\right|-\left|B_{r-\varepsilon \tau_{W}}^{\Phi^{\circ}}\left(x_{0}+\varepsilon \gamma\left(y_{0}-x_{0}\right)\right)\right| \\
& +n \kappa_{\Phi} \int_{r-\varepsilon \tau_{W}}^{r+\varepsilon \tau_{W}} z\left(\frac{\rho-r}{\varepsilon}\right) \rho^{n-1} d \rho-m \\
= & |\Omega|-m-\kappa_{\Phi}\left(\left(r+\varepsilon \tau_{W}\right)^{n}+\left(r-\varepsilon \tau_{W}\right)^{n}-n \varepsilon \int_{-\tau_{W}}^{\tau_{W}} z(t)(r+\varepsilon t)^{n-1} d t\right) .
\end{aligned}
$$

Since $z$ is odd, by (2.19) there exists a constant $M>0$ such that

$$
\left|\omega_{\varepsilon}\right| \leq M \varepsilon^{2}
$$

for $\varepsilon>0$ sufficiently small.

We now correct $\hat{u}_{\varepsilon}$ in order to satisfy the mass constraint in (1.2). We fix $\varphi \in C_{c}^{\infty}\left(\mathbb{R}^{n}\right)$ with support contained in $B_{r / 2}^{\Phi^{\circ}}\left(x_{0}\right)$ and

$$
\int_{\mathbb{R}^{n}} \varphi(x) d x=1,
$$


and we define

$$
u_{\varepsilon}(x):=\hat{u}_{\varepsilon}(x)-\omega_{\varepsilon} \varphi(x) .
$$

Taking into account the definition of $\omega_{\varepsilon}$, we find that by (4.5), $u_{\varepsilon}$ satisfies the mass constraint in (1.2). Since $B_{r / 2}^{\Phi^{\circ}}\left(x_{0}\right) \subset B_{r-\varepsilon \tau_{W}}^{\Phi^{\circ}}\left(x_{0}+\varepsilon \gamma\left(y_{0}-x_{0}\right)\right)$, the support of $\varphi$ is contained in $\left\{\hat{u}_{\varepsilon}=-1\right\}$. Hence $u_{\varepsilon}$ still satisfies the boundary condition in (1.2) for $\varepsilon>0$ sufficiently small, and

$$
\begin{aligned}
\int_{\Omega} W\left(u_{\varepsilon}(x)\right) d x & =\int_{\left\{\hat{u}_{\varepsilon} \neq-1\right\}} W\left(\hat{u}_{\varepsilon}(x)\right) d x+\int_{\left\{\hat{u}_{\varepsilon}=-1\right\}} W\left(-1-\omega_{\varepsilon} \varphi(x)\right) d x \\
& =\int_{\Omega} W\left(\hat{u}_{\varepsilon}(x)\right) d x+\int_{\Omega} W\left(-1-\omega_{\varepsilon} \varphi(x)\right) d x,
\end{aligned}
$$

where the last equality follows from the fact that $W(-1)=0$. Since the supports of $\nabla \hat{u}_{\varepsilon}$ and $\nabla \varphi$ are disjoint, the previous equality implies that

$$
\mathcal{E}_{\varepsilon}\left(u_{\varepsilon}\right)=\mathcal{E}_{\varepsilon}\left(\hat{u}_{\varepsilon}\right)+\mathcal{E}_{\varepsilon}\left(-1-\omega_{\varepsilon} \varphi\right) .
$$

By (2.16), (4.3), and (4.6) we have

$$
\Phi\left(\nabla \hat{u}_{\varepsilon}(x)\right)=\frac{1}{\varepsilon} z^{\prime}\left(\frac{\Phi^{\circ}\left(x-x_{0}-\varepsilon \gamma\left(y_{0}-x_{0}\right)\right)-r}{\varepsilon}\right)
$$

for a.e. $x \in \Omega$. Since $z(t)= \pm 1$ for $\pm t \geq \tau_{W}$, by (2.15) and by the equality $W( \pm 1)=0$ we obtain

$$
\begin{aligned}
\mathcal{E}_{\varepsilon}\left(\hat{u}_{\varepsilon}\right) & =\frac{n \kappa_{\Phi}}{\varepsilon} \int_{r-\varepsilon \tau_{W}}^{r+\varepsilon \tau_{W}}\left(W\left(z\left(\frac{\rho-r}{\varepsilon}\right)\right)+\left|z^{\prime}\left(\frac{\rho-r}{\varepsilon}\right)\right|^{2}\right) \rho^{n-1} d \rho \\
& =n \kappa_{\Phi} \int_{-\tau_{W}}^{\tau_{W}}\left(W(z(t))+\left|z^{\prime}(t)\right|^{2}\right)(r+\varepsilon t)^{n-1} d t \\
& =n \kappa_{\Phi} c_{W} r^{n-1}+O\left(\varepsilon^{2}\right),
\end{aligned}
$$

where we used the change of variables $t=\frac{\rho-r}{\varepsilon}$ and (2.7), and in the last equality we used (2.7), taking into account once again the fact that $t \mapsto(W(z(t))+$ $\left.\left|z^{\prime}(t)\right|^{2}\right) t$ is odd.

On the other hand, by (2.4) and (2.10),

$$
\mathcal{E}_{\varepsilon}\left(-1-\omega_{\varepsilon} \varphi\right) \leq \frac{\left|\omega_{\varepsilon}\right|^{\beta}}{\varepsilon} \int_{\Omega}|\varphi|^{\beta} d x+C_{\Phi} \varepsilon\left|\omega_{\varepsilon}\right|^{2} \int_{\Omega}|\nabla \varphi|^{2} d x .
$$

From (4.7), (4.8) and (4.9) we get

$$
\begin{aligned}
& \frac{\mathcal{E}_{\varepsilon}\left(u_{\varepsilon}\right)-n \kappa_{\Phi} c_{W} r^{n-1}}{\varepsilon} \\
& \quad \leq \frac{\left|\omega_{\varepsilon}\right|^{\beta}}{\varepsilon^{2}} \int_{\Omega}|\varphi|^{\beta} d x+C_{\Phi}\left|\omega_{\varepsilon}\right|^{2} \int_{\Omega}|\nabla \varphi|^{2} d x+O(\varepsilon) .
\end{aligned}
$$

Recalling that $\beta>1$, from (4.4) we obtain (4.1). 


\section{Acknowledgments}

The authors warmly thank the Center for Nonlinear Analysis (NSF Grant No. DMS-0635983), where part of this research was carried out. The research of I. Fonseca was partially funded by the National Science Foundation under Grant No. DMS-0905778 and that of G. Leoni under Grant No. DMS-1007989. I. Fonseca and G. Leoni also acknowledge support of the National Science Foundation under the PIRE Grant No. OISE-0967140. The research of G. Dal Maso was also supported by by the Italian Ministry of Education, University, and Research under the Project "Variational Problems with Multiple Scales" 2008 and by the European Research Council under Grant No. 290888 "Quasistatic and Dynamic Evolution Problems in Plasticity and Fracture". The authors wish to thank Matteo Focardi for several discussions on the subject of this paper and Michael Goldman, who called their attention to reference [1]. The results of this paper led to a dramatic simplification in the proof of the $\Gamma$-limsup inequality and were crucial in the proof of the $\Gamma$-liminf inequality in the anisotropic case.

\section{References}

[1] A. Alvino, V. Ferone, G. Trombetti, P.-L. Lions, Convex symmetrization and applications. Ann. Inst. H. Poincaré Anal. Non Linéaire 14 (1997), no. $2,275-293$.

[2] G. Anzellotti, S. Baldo, Asymptotic development by $\Gamma$-convergence. Appl. Math. Optim. 27 (1993), no. 2, 105-123.

[3] G. Anzellotti, S. Baldo, G. Orlandi, Г-asymptotic developments, the CahnHilliard functional, and curvatures. J. Math. Anal. Appl. 197 (1996), no. $3,908-924$.

[4] A.C. Barroso, I. Fonseca, Anisotropic singular perturbations-the vectorial case. Proc. Roy. Soc. Edinburgh Sect. A 124 (1994), no. 3, 527-571.

[5] G. Bellettini, A. Nayam, M. Novaga, Г-type estimates for the onedimensional Allen-Cahn's action. Submitted.

[6] G. Bouchitté, Singular perturbations of variational problems arising from a two-phase transition model. Appl. Math. Optim. 21 (1990), no. 3, 289-314.

[7] A. Braides, Approximation of Free-Discontinuity Problems. Lecture Notes in Mathematics 1694, Springer Verlag, Berlin, 1998.

[8] A. Braides, $\Gamma$-convergence for beginners. Oxford Lecture Series in Mathematics and its Applications, 22. Oxford University Press, Oxford, 2002.

[9] J. Carr, M.E. Gurtin, and M. Slemrod, Structured phase transitions on a finite interval. Arch. Rat. Mech. Anal. 86 (1984), no. 4, 317-351. 
[10] G. Dal Maso, An Introduction to $\Gamma$-Convergence, Progress in Nonlinear Differential Equations and their Applications. 8. Basel: Birkhäuser, 1993.

[11] G. Dal Maso, R. Musina, An approach to the thin obstacle problem for variational functionals depending on vector valued functions. Comm. Partial Differential Equations 14 (1989), no. 12, 1717-1743.

[12] I. Fonseca, The Wulff theorem revisited. Proc. Roy. Soc. London Ser. A 432 (1991), no. 1884, 125-145.

[13] I. Fonseca, S. Müller, A uniqueness proof for the Wulff theorem. Proc. Roy. Soc. Edinburgh Sect. A 119 (1991), no. 1-2, 125-136.

[14] I. Fonseca, L. Tartar, The gradient theory of phase transitions for systems with two potential wells. Proc. Roy. Soc. Edinburgh Sect. A 111 (1989), no. 1-2, 89-102.

[15] M.E. Gurtin, Some results and conjectures in the gradient theory of phase transitions. IMA, preprint 156 (1985).

[16] S. Lay, Convex Sets and Their Applications. Revised reprint of the 1982 original. Robert E. Krieger Publishing Co., Inc., Malabar, FL, 1992.

[17] G. Leoni, A first course in Sobolev spaces. Graduate Studies in Mathematics, 105. American Mathematical Society, Providence, RI, 2009

[18] G. Leoni, R. Murray, Second order $\Gamma$-limit for the Cahn-Hilliard functional. In preparation.

[19] S. Luckhaus, L. Modica, The Gibbs-Thompson relation within the gradient theory of phase transitions. Arch. Rational Mech. Anal. 107 (1989), no. 1, $71-83$.

[20] L. Modica, S. Mortola, Un esempio di $\Gamma^{-}$-convergenza. Boll. Un. Mat. Ital. B (5) 14 (1977), no. 1, 285-299.

[21] L. Modica, The gradient theory of phase transitions and the minimal interface criterion, Arch. Rational Mech. Anal. 98 (1987), no. 2, 123-142.

[22] F. Morgan, The cone over the Clifford torus in $\mathbf{R}^{4}$ is $\Phi$-minimizing. Math. Ann. 289 (1991), no. 2, 341-354.

[23] N.C. Owen, P. Sternberg, Nonconvex variational problems with anisotropic perturbations. Nonlinear Anal. 16 (1991), no. 7-8, 705-719.

[24] R. T. Rockafellar, Convex analysis. Reprint of the 1970 original. Princeton Landmarks in Mathematics. Princeton Paperbacks. Princeton University Press, Princeton, NJ, 1997.

[25] R. Schneider, Convex bodies: the Brunn-Minkowski theory. Encyclopedia of Mathematics and its Applications, 44. Cambridge University Press, Cambridge, 1993. 
[26] P. Sternberg, The effect of a singular perturbation on nonconvex variational problems, Arch. Rational Mech. Anal. 101 (1988), no. 3, 209-260.

[27] J.E. Taylor, Existence and structure of solutions to a class of nonelliptic variational problems. Symposia Mathematica, Vol. XIV (Convegno di Teoria Geometrica dell'Integrazione e Varietà Minimali, INDAM, Roma, Maggio 1973), 499-508. Academic Press, London, 1974.

[28] J.E. Taylor, Unique structure of solutions to a class of nonelliptic variational problems. (Proc. Sympos. Pure. Math., Vol. XXVII, Stanford Univ., Stanford, Calif., 1973), Part 1, 419-427. Amer. Math. Soc., Providence, R.I., 1975.

[29] J.E. Taylor, Crystalline variational problems. Bull. Amer. Math. Soc. 84 (1978), no. 4, 568-588.

[30] G. Wulff, Zur Frage der Geschwindigkeit des Wachstums und der Auflösung der Kristallflächen. Z. Kristallogr. 34 (1901), 449-530. 